Dedicated to the blessed memory of our teachers and senior colleagues,

Alexey Borisovich Shabat

and Ravil Islamovich Yamilov

doi:10.13108/2021-13-2-41

\title{
CHARACTERISTIC ALGEBRAS AND INTEGRABLE EXPONENTIAL SYSTEMS
}

\section{D.V. MILLIONSHCHIKOV, S.V. SMIRNOV}

\begin{abstract}
In the present paper we study characteristic algebras for exponential systems corresponding to degenerate Cartan matrices. These systems generalize hyperbolic sineGordon and Tzitzeica equations well-known in the theory of integrable systems. For such systems, corresponding to Cartan matrices of rank 2, we describe explicitly characteristic algebras in terms of generators and relations and we prove that they have linear growth. We study the relations between the higher symmetries of these systems and the structure of their characteristic algebras. We describe completely the higher symmetries of exponential systems corresponding to the Cartan matrix of affine Lie algebra $A_{2}^{(1)}$. We also obtain partial results on symmetries of such systems corresponding to other degenerate Cartan matrices of rank 2. We propose a conjecture on the structure of higher symmetries of arbitrary exponential system corresponding to a degenerate Cartan matrix. We study an interesting combinatorics related to an operator generating a characteristic algebra in the simplest case for a Darboux integrable Liouville equation. The found combinatorial properties can be very useful for proving the aforementioned conjecture on the structure of higher symmetries. Moreover, in the present paper we give a rigorous meaning to the concept of a characteristic algebra of a hyperbolic system used for a long time in the literature. We do this by means of the notion of Lie-Rinehart algebra and at the examples we demonstrate that such formalization is indeed needed.
\end{abstract}

Keywords: characteristic algebra, higher symmetry, Liouville equation, exponential system.

Mathematics Subject Classification: 37K10, 37K30, 17B67, 17B70

\section{INTRODUCTION}

In 1981, A.B. Shabat and R.I. Yamilov published preprint [1], in which they considered socalled exponential type systems, that is, system of hyperbolic partial differential equations of form

$$
w_{x y}^{j}=\exp \left(\sum_{k=1}^{r} a_{j k} w^{k}\right), \quad j=1,2, \ldots, r,
$$

where $a_{j k}$ are constant coefficients and the functions $w^{j}$ depend on variables $x$ and $y$. It is easy to confirm that if the matrix $M=\left(a_{j k}\right)$ is a Cartan matrix of a simple Lie algebra of series $A$,

D.V. Millionshchikov, S.V. Smirnov, Characteristic algebras and integrable exponential SYSTEMS.

(C) Millionshchikov D.V., Smirnov S.V. 2021.

The research by S.V. Smirnov (Sections 6 7) is supported by Russian Science Foundation, project no. 2011-20214.

Submitted April 21, 2021. 
then corresponding system 1.1 is reduced to a two-dimensional Toda chain

$$
q_{j, x y}=\exp \left(q_{j+1}-q_{j}\right)-\exp \left(q_{j}-q_{j-1}\right), \quad j=0,1, \ldots, r,
$$

with trivial boundary conditions $q_{-1} \equiv \infty, q_{r+1} \equiv-\infty$.

The study of systems of form (1.1) in [1] was based on investigating characteristic algebras, i.e., Lie algebras generated by the operators $D_{y}$ of total differentiation with respect to the variable $y$ by virtue of the system and $\frac{\partial}{\partial w^{j}}$. Shabat and Yamilov formulated two important conjectures on the integrability of exponential type systems. The first conjecture is that system (1.1) is Darboux integrable, i.e., it admits complete sets of independent characteristic integrals in both variables, if and only if it is a direct sum of several exponential type systems, the matrix $M$ of which are Cartan matrices of simple Lie algebras. The second conjecture says that systems of form (1.1), not Darboux integrable, but integrable by the inverse problem method, correspond to slowly growing characteristic algebras. Despite the fact that the proof of the main classification theorem in [1] contained some gaps that have not been eliminated up to this day (which, apparently, was the reason that this text remained in the preprint status), this work served as a starting point for a large number of studies and publications on exponential systems. In particular, in [2, integrable exponential type systems with Cartan matrices of size 2 were studied, and in [3], systems corresponding to non-degenerate Cartan matrices and, in particular, systems corresponding to Lie algebras of the series $A$, were integrated explicitly. We also mention papers [4]-[6] by the Ufa school, in which reductions of exponential type systems and their connection with higher symmetries were studied, and a series of papers [7]-[17], in which discrete analogs of exponential type systems were studied.

Although the ideas related to the concept of characteristic algebra were implicitly used by Goursat [18] in his attempts to classify Darboux integrable scalar hyperbolic equations, the corresponding definition was introduced only in the last quarter of the twentieth century in [19]. Later the concept of characteristic algebra was widely used in studying Darboux integrable discrete and semi-discrete equations in works [20]-[30] of the Ufa school. A detailed survey of the applications of characteristic algebras to the study of nonlinear equations is contained in book [31]. The symmetries of the Klein-Gordon equations were studied in paper [32].

Characteristic algebras of integrable hyperbolic equations provide examples of graded infinitedimensional Lie algebras of linear growth. In paper [21], the characteristic algebra of the sineGordon equation, having a growth rate of $\frac{3}{2}$ was studied, and in [25], there was studied the characteristic algebra of the Tzitzeica equation growing at an average rate of $\frac{4}{3}$. In papers [33], [34] infinite linear bases for these Lie algebras were constructed and it was shown that they are isomorphic to the nonnegative part of the loop algebra $\mathfrak{s l}(2, \mathbb{C}) \otimes \mathbb{C}\left[t, t^{-1}\right]$ and to the twisted loop algebra of the simple Lie algebra $\mathfrak{s l}(3, \mathbb{C})$, respectively.

The present work is aimed at developing some of ideas expressed in preprint [1] and on a progress in proving the conjectures expressed there. We have several goals. First, we are going to give a due algebraic rigor to the concept of characteristic algebra (examples will be given showing that this is absolutely necessary in the discrete case): we will show that it is natural to treat these objects not as Lie algebras (or Lie rings), but as Lie-Rinehart algebras. Second, we investigate an interesting combinatorics related to the Liouville equation, the simplest representative of the class of exponential systems corresponding to Cartan matrices. And, thirdly, we explicitly describe characteristic algebras of exponential type (1.1) with degenerate Cartan matrices of rank 2 and describe higher symmetries for some of them. As in the case of systems of rank 1, which can be reduced to the sine-Gordon and Tzizeica equations, the characteristic algebras of these systems have linear growth.

In Section 2 we provide a brief survey of known results on the integrability of exponential type systems corresponding to the Cartan matrices. In Section 3 we introduce the concept of a LieRinehart algebra, describe the structure of the characteristic algebras of the sine-Gordon and 
Tzizeica equations, and adduce an example showing that it is appropriate to treat characteristic algebras exactly as Lie-Rinehart algebras. Section 4 is devoted to describing the notion of growth for infinite-dimensional Lie algebras. In Section 5 we study an interesting combinatorics related to the characteristic integrals and symmetries of the Liouville equation. Section 6 is devoted to an explicit description of exponential systems corresponding to degenerate Cartan matrices of rank 2 in terms of the generators and relations of characteristic algebras. In Section 7 we describe the symmetry hierarchy for the system corresponding to the affine Cartan matrix $A_{2}^{(1)}$ and gives some partial results on higher symmetries of systems corresponding to other degenerate Cartan matrices of rank 2.

\section{EXPONENTIAL TYPE SYSTEM AND CARTAN MATRICES}

We consider a hyperbolic system of partial differential equations of exponential type being a multidimensional generalization of the classical Liouville equation

$$
w_{x y}^{i}=e^{\rho_{i}}, \quad \rho_{j}=a_{i 1} w^{1}+\cdots+a_{i r} w^{r}, \quad i=1, \ldots, r .
$$

Leznov [3] showed that if the matrix of the coefficients $M=\left(a_{i j}\right)$ is a nondegenerate Cartan matrix, then the corresponding exponential hyperbolic system (2.1) is Darboux integrable. In his proof [3], for the Cartan matrix from the $A$ series, an exact solution of the system was found explicitly and it depended on $2 r$ arbitrary functions, that is, there was constructed a multidimensional generalization of the classical solution of the one-dimensional Liouville equation $u_{x y}=e^{u}$. Later, in preprint [1], a conjecture was made that the main result of [3] could be extended to the case of an arbitrary Cartan matrix $M$, possibly degenerate, using the method of the inverse scattering problem. The case $r=2$ was completely solved in [1, 2]:

$$
\left\{\begin{array}{l}
w_{x y}^{1}=e^{\left(a_{11} w^{1}+a_{12} w^{2}\right)} \\
w_{x y}^{2}=e^{\left(a_{21} w^{1}+a_{22} w^{2}\right)},
\end{array} \quad M=\left(\begin{array}{ll}
a_{11} & a_{12} \\
a_{21} & a_{22}
\end{array}\right) .\right.
$$

It was shown [1, 2] that for two degenerate Cartan matrices

$$
M_{1}=\left(\begin{array}{cc}
2 & -2 \\
-2 & 2
\end{array}\right), \quad M_{2}=\left(\begin{array}{cc}
2 & -4 \\
-1 & 2
\end{array}\right)
$$

corresponding exponential systems $(2.2)$ are integrable by the inverse scattering problem method. The characteristic Lie algebras of these equations, which will be discussed in the next section, are isomorphic to some Lie subalgebras in the Lie algebras $A_{1}^{(1)}, A_{2}^{(2)}$. Exponential systems 2.2 corresponding to non-degenerate Cartan matrices of size $2 \times 2$

$$
\left(\begin{array}{ll}
2 & 0 \\
0 & 2
\end{array}\right), \quad\left(\begin{array}{cc}
2 & -1 \\
-1 & 2
\end{array}\right), \quad\left(\begin{array}{cc}
2 & -2 \\
-1 & 2
\end{array}\right), \quad\left(\begin{array}{cc}
2 & -3 \\
-1 & 2
\end{array}\right)
$$

of semi-simple Lie algebras $A_{1} \oplus A_{1}, A_{2}, C_{2}, G_{2}$ are integrable in an explicit form.

Now we consider a degenerate Cartan matrix $M=\left(a_{i j}\right)$. Its last row $l_{r}$ can be represented as a linear combination of the first $r-1$ rows:

$$
l_{r}=\lambda_{1} l_{1}+\cdots+\lambda_{r-1} l_{r-1} .
$$

We introduce new variables

$$
u^{i}=a_{i 1} w^{1}+\cdots+a_{i r} w^{r}, \quad i=1,2, \ldots r-1 .
$$

Then system (2.1) can be rewritten as

$$
u_{x y}^{i}=a_{i 1} e^{u_{1}}+a_{i 2} e^{u_{2}}+\cdots+a_{i r-1} e^{u_{r-1}}+a_{i r} e^{\lambda_{1} u_{1}+\cdots+\lambda_{r-1} u_{r-1}}, \quad i=1,2, \ldots, r-1 .
$$

Thus, for degenerate Cartan matrices of size $r$, the corresponding exponential system is reduced to a system consisting of $r-1$ hyperbolic equations. In particular, a degenerate Cartan matrix of size 2 leads to the scalar sine-Gordon and Tzizeica equations. In Section 6 we study the 
systems to which the exponential systems corresponding to degenerate Cartan matrices of size 3 are reduced.

\section{Characteristic LiE-Rinehart Algebras}

Let us recall the construction of one generalization of the notion of Lie algebra known in the literature as the Lie-Rinehart algebra.

Definition 3.1 ([35]). Let $R$ be a unital associative ring and $A$ be a commutative $R$-algebra. The pair $(A, \mathcal{L})$ is called Lie-Rinehart algebra if

1) $\mathcal{L}$ is a Lie algebra over $R$ acting on $A$ by left differentiations, i.e.,

$$
X(a b)=X(a) b+a X(b) \quad \text { for all } a, b \in A, X \in \mathcal{L} ;
$$

2) Lie algebra $\mathcal{L}$ is an $A$-module.

The pair $(A, \mathcal{L})$ should satisfy the following compatibility conditions:

$$
\begin{aligned}
& {[X, a Y]=X(a) Y+a[X, Y] \quad \text { for all } \quad X, Y \in \mathcal{L}, a \in A ;} \\
& (a X)(b)=a(X(b)) \text { for all } a, b \in A, X \in \mathcal{L} .
\end{aligned}
$$

A morphism $\varphi:(A, \mathcal{L}) \rightarrow(A, \tilde{\mathcal{L}})$ of Lie-Rineharts algebras $(A, \mathcal{L})$ and $(A, \tilde{\mathcal{L}})$ is a morphism $\varphi: \mathcal{L} \rightarrow \tilde{\mathcal{L}}$ of Lie algebras over $R$ compatible with the action $\mathcal{L}$ over $A$.

The concept of a Lie-Rinehart algebra turns out to arise naturally in the theory of characteristic Lie algebras of hyperbolic systems. As a basic example, let us consider the characteristic Lie algebra $\chi(\sinh (u))$ of the sine-Gordon equation.

It was established in [33], [34] that in the characteristic Lie algebra

$$
\chi(\sinh (u))=\mathcal{L} i e\left(X_{0}, X_{1}\right)
$$

we can choose an infinite basis $X_{0}, X_{1}, X_{2}, X_{3}, \ldots$, such that

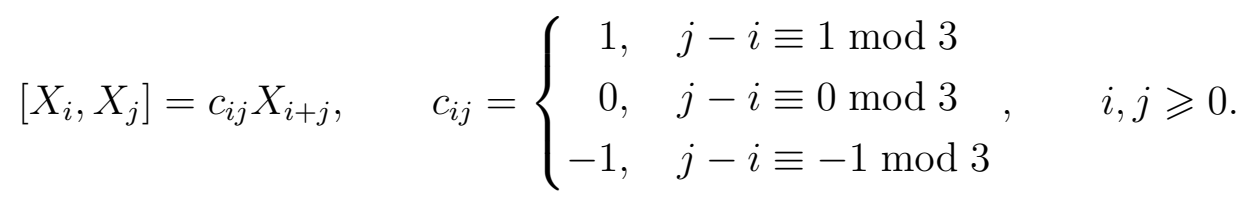

Structure relations (3.1) coincide with the canonical relations for the basic elements of the algebra of nonnegative loops $\mathcal{L}(\mathfrak{s l}(2, \mathbb{C}))^{\leqslant 0}$ over $\mathfrak{s l}(2, \mathbb{C})$ or, in other terms, with the nonnegative part of the affine Kac-Moody algebra $A_{1}^{(1)}$.

The characteristic Lie algebra $\chi(\sinh (u))$ is a Lie algebra, but in the proof of its commutation relations the structure of some auxiliary Lie-Rinehart algebra is essentially employed. It should be noted that in monograph [31], as well as in a series of papers [25]-[28] and in others, the term Lie ring was used. This choice was not very successful, and now in the literature a correct, in our opinion, term Lie-Reinhart algebra is used more and more often [30].

We recall some known constructions. We consider the Klein-Gordon equation $u_{x y}=f(u)$ obtained from the classical equation

$$
u_{t t}-u_{z z}=f(u)
$$

by the change of variables $x=\frac{z+t}{2}, y=\frac{z-t}{2}$.

We define a composite function $g(x, y)$ by means of a function $g$ of many variables and some solution $u(x, y)$ of the Klein-Gordon equation

$$
u=u(x, y), \quad g(x, y)=g\left(u, u_{x}, u_{x x}, u_{x x x}, \ldots\right)=g\left(u, u_{1}, u_{2}, u_{3}, \ldots\right),
$$

where we have used the notations

$$
u_{1}=u_{x}, \quad u_{2}=u_{x x}, \quad u_{3}=u_{x x x}, \quad \ldots
$$


A classical construction is as follows: while calculating a partial derivative $\frac{\partial g}{\partial x}$ of a composite function

$$
\frac{\partial g}{\partial x}=\frac{\partial u}{\partial x} \frac{\partial g}{\partial u}+\frac{\partial u_{1}}{\partial x} \frac{\partial g}{\partial u_{1}}+\cdots=u_{1} \frac{\partial g}{\partial u}+u_{2} \frac{\partial g}{\partial u_{1}}+\ldots
$$

an operator $D=\frac{\partial}{\partial x}$ of a partial differentiation in $x$ arises:

$$
D=u_{1} \frac{\partial}{\partial u}+u_{2} \frac{\partial}{\partial u_{1}}+u_{3} \frac{\partial}{\partial u_{2}}+\cdots+u_{k+1} \frac{\partial}{\partial u_{k}}+\ldots
$$

Definition 3.2. The polynomial $F\left(u, u_{x}, u_{x x}, u_{x x x}, \ldots\right)=F\left(u, u_{1}, u_{2}, u_{3}, \ldots\right)$ is called $y$ integral of the Klein-Gordon equation if $\frac{\partial F}{\partial y}=0$.

We consider

$$
\begin{aligned}
\frac{\partial}{\partial y} F\left(u, u_{1}, u_{2}, \ldots\right) & =u_{y} \frac{\partial F}{\partial u}+u_{x y} \frac{\partial F}{\partial u_{1}}+u_{x x y} \frac{\partial F}{\partial u_{2}}+\ldots \\
& =\underbrace{u_{y} \frac{\partial F}{\partial u}}_{=0}+\underbrace{f \frac{\partial F}{\partial u_{1}}+\frac{\partial f}{\partial x} \frac{\partial F}{\partial u_{2}}+\ldots}_{=0}
\end{aligned}
$$

The polynomial $F$ is a $y$-interal if the following conditions are satisfied

$$
\frac{\partial F}{\partial u}=0, \quad X_{f}(F)=f \frac{\partial F}{\partial u_{1}}+D(f) \frac{\partial F}{\partial u_{2}}+D^{2}(f) \frac{\partial F}{\partial u_{2}}+\cdots=0 .
$$

We recall that a characteristic algebra $\chi(\sinh (u))$ of the sine-Gordon equation is the Lie algebra $\mathcal{L} i e\left(X_{0}, X_{\sinh u}\right)$ generated by two differential operators $X_{0}, X_{\sinh u}$, where

$$
X_{0}=\frac{\partial}{\partial u}, \quad X_{\sinh u}=\sum_{n=1}^{+\infty} D_{x}^{n-1}(\sinh u) \frac{\partial}{\partial u_{n}}
$$

and $D_{y}=X_{\sinh u}+X_{0}$. We shall construct a required basis in $\chi(\sinh (u))$ step-by-step by considering all commutators of higher order of generators $X_{0}, X_{\sinh u}$.

At the first step we fix a linear $\operatorname{span}\left\langle X_{0}, X_{\sinh u}, Y_{1}\right\rangle$, where $Y_{1}=\left[X_{0}, X_{\sinh u}\right]$ and we consider another basis in this span:

$$
X_{0}, \quad X_{1}=X_{\sinh u}+Y_{1}, \quad X_{2}=X_{\sinh u}-Y_{1} .
$$

New operators can be written as follows

$$
X_{1}=\sum_{n=1}^{+\infty} D_{x}^{n-1}\left(e^{u}\right) \frac{\partial}{\partial u_{n}}, \quad X_{2}=-\sum_{n=1}^{+\infty} D_{x}^{n-1}\left(e^{-u}\right) \frac{\partial}{\partial u_{n}} .
$$

We observe that these operators can be expressed in terms of Bell polynomials $B_{n}\left(u_{1}, \ldots, u_{n}\right)$, which we will discussed more detail in Section 5 .

$$
X_{1}=e^{u} \sum_{n=1}^{+\infty} B_{n-1}\left(u_{1}, \ldots, u_{n-1}\right) \frac{\partial}{\partial u_{n}}, \quad X_{2}=-e^{-u} \sum_{n=1}^{+\infty} B_{n-1}\left(-u_{1}, \ldots,-u_{n-1}\right) \frac{\partial}{\partial u_{n}} .
$$

The first idea of the proof [33], 34] is to represent the characteristic Lie algebra $\chi(\sinh (u))$ as the Lie algebra $\mathcal{L} i e\left(X_{0}, X_{1}, X_{2}\right)$ generated not by two, but by three elements $X_{0}, X_{1}, X_{2}$. The advantage of the representation $\chi(\sinh (u))=\mathcal{L} i e\left(X_{0}, X_{1}, X_{2}\right)$ is the following two commutation relations:

$$
\left[X_{0}, X_{1}\right]=X_{1}, \quad\left[X_{0}, X_{2}\right]=-X_{2},
$$

which mean that the vectors $X_{1}, X_{2}$ are eigenvectors for the operator ad $X_{0}$ and the same is true for all their higher commutators. Exactly this fact will be key in all calculations. 
The second idea of the proof from [33], [34] is to observe that commutation relations (3.1) can be found without obtaining explicit formulae for the operators $X_{3}, X_{4}, X_{5}, \ldots$ This idea is not original and is based on a well-known elementary lemma.

Lemma 3.1 ([31]). Let the differential operator

$$
X=\sum_{i=1}^{+\infty} P_{i} \frac{\partial}{\partial u_{i}}, \quad P_{i}=P_{i}\left(u, u_{1}, \ldots, u_{n}, \ldots\right),
$$

commute with the operator $D$. Then $X=0$.

We stress that the identity $\left[D, X_{0}\right]=0$ does not contradict to the previous lemma in the identity since the operator $X_{0}=\frac{\partial}{\partial u}$ does not have the form specified in the assumptions of Lemma 3.1.

It is easy to check the commutation relations

$$
\left[D, X_{1}\right]=-e^{u} X_{0}, \quad\left[D, X_{2}\right]=e^{-u} X_{0} .
$$

The appearance of the functional factors $e^{u}, e^{-u}$ in the right hand sides of these formulas indicates the need to consider Lie-Rinehart algebras instead of ordinary Lie algebras.

As an example, we provide one more commutation relation, in which the functional factors $e^{u}, e^{-u}$ appear as well:

$$
\left[D, X_{3}\right]=\left[D,\left[X_{1}, X_{2}\right]\right]=-\left[e^{u} X_{0}, X_{2}\right]+\left[X_{1}, e^{-u} X_{0}\right]=e^{u} X_{2}-e^{-u} X_{1} .
$$

What can we say about the Lie algebra $\mathcal{L} i e\left(D, X_{0}, X_{1}, X_{2}\right)$ generated by the operators $D$, $X_{0}, X_{1}, X_{2}$ ? Exactly this algebra should serve as the Lie subalgebra in the Lie-Rinehart algebra $(A, \mathcal{L})$, where $A$ denotes a commutative algebra of quasipolynomials of the form

$$
A=\left\{\sum_{k=-n}^{m} e^{k u} P_{k}, \quad P_{k} \in \mathbb{C}\left[u_{1}, u_{2}, u_{3}, \ldots\right], \quad n, m \in \mathbb{N}\right\},
$$

and the Lie algebra $\mathcal{L}$ is the algebra of quasipolynomial differential operators

$$
X=f_{0} \frac{\partial}{\partial u}+f_{1} \frac{\partial}{\partial u^{1}}+f_{2} \frac{\partial}{\partial u^{2}}+\ldots, \quad f_{i} \in A, \quad i \in \mathbb{N} .
$$

We observe that

$$
\left[D,-e^{u} X_{0}\right]=-D\left(e^{u}\right) X_{0}=-e^{u} u_{1} X_{0}, \quad\left[D, e^{-u} X_{0}\right]=-e^{-u} u_{1} X_{0} .
$$

Continuing this process recurrently, we see that the Lie algebra $\mathcal{L} i e\left(D, X_{0}, X_{1}, X_{2}\right)$ also contains operators with coefficients in the form of quasipolynomials of arbitrary weights

$$
-e^{u} B_{n}\left(u_{1}, \ldots, u_{n}\right) X_{0}, \quad e^{-u} B_{k}\left(-u_{1}, \ldots,-u_{n}\right) X_{0}, \quad n, k \in \mathbb{N} .
$$

It would be interesting to study $\mathcal{L} i e\left(D, X_{0}, X_{1}, X_{2}\right)$ from the point of view of infinitedimensional $\mathbb{Z}$-graded Lie algebras. It seems that it should be isomorphic to one of known algebras of such type.

However, the main aim of all our calculations is the proof of commutation relations (3.1):

$$
\left[D, X_{4}\right]=-\left[\left[D, X_{1}\right], X_{3}\right]-\left[X_{1},\left[D, X_{3}\right]\right]=\left[e^{u} X_{0}, X_{3}\right]-\left[X_{1}, e^{u} X_{2}+e^{-u} X_{1}\right]=-e^{u} X_{3},
$$

where $X_{4}=-\left[X_{1}, X_{3}\right]$.

Let us prove that $\left[X_{1}, X_{4}\right]=0$. In order to do this, we calculate a double commutator $\left[D,\left[X_{1}, X_{4}\right]\right]=\left[\left[D, X_{1}\right], X_{4}\right]+\left[X_{1},\left[D, X_{4}\right]\right]=-\left[e^{u} X_{0}, X_{4}\right]-\left[X_{1}, e^{u} X_{3}\right]=-e^{u} X_{4}+e^{u} X_{4}=0$.

In the same way we prove [34] that $\left[X_{2}, X_{5}\right]=0$. 
Then we define inductively all operators of the canonical infinite basis for the characteristic Lie algebra $\chi(\sinh (u))$ by the formulae

$$
X_{3 k+1}=-\left[X_{1}, X_{3 k}\right], \quad X_{3 k+2}=\left[X_{2}, X_{3 k}\right], \quad X_{3 k+3}=\left[X_{1}, X_{3 k+2}\right], \quad k \geqslant 1 .
$$

And for these operators, by induction, we prove commutation relations (3.1).

Following paper [2], another way of algebraic arguing can be proposed. Its matter is that the functional factors $e^{\lambda u}$ are "removed" from the basis operators, in other words, we introduce new operators

$$
L_{3 k+1}=e^{-u} X_{3 k+1}^{\prime}, \quad L_{3 k+2}=e^{u} X_{3 k+2}^{\prime}, \quad L_{3 k+3}=X_{3 k+3}^{\prime} .
$$

It is obvious that the new differential operators $L_{i}$ satisfy the same commutation relations (3.1) as the operators $X_{i}$ do, but at the same time they are polynomial, i.e., they are the differentiation operators of the algebra of polynomials $\mathbb{C}\left[u_{1}, u_{2}, \ldots, u_{p}, \ldots\right]$.

The commutation relations involving the operator $D$ change their form. Let us write down two main relations:

$$
\left[D, L_{1}\right]=-u_{1} L_{1}, \quad\left[D, L_{2}\right]=u_{1} L_{2} .
$$

We arrive at the construction of so-called polynomial Lie algebra proposed by Buchstaber [36]. A polynomial Lie algebra in Buchstaber sense is the Lie-Rinehart algebra in the situation when the algebra $A$ is some algebra of polynomials, usually on finitely many variables, and at the same time, two additional important conditions are to be satisfied:

1) the Lie algebra $\mathcal{L}$ mentioned in the definition should be a free module, usually finitedimensional, over the algebra $A$;

2) the Lie algebra $\mathcal{L}$ and the polynomial algebra $A$ should be graded and the gradings should be compatible in a natural sense.

As it was shown in [37] at the example of $\chi(\sinh (u))$, the study of characteristic Lie algebras leads one to the need to consider polynomial Lie algebras $A$ in infinitely many variables, and the Lie algebras are modules of a countable rank over the polynomial algebra $A$.

Now let us define $\mathbb{Z}$-gradings of the polynomial algebra $A=\mathbb{C}\left[u_{1}, u_{2}, \ldots, u_{p}, \ldots\right]$ and of the Lie algebra $\mathcal{L}$ for the characteristic Lie algebra of the sine-Gordon equation. We introduce weights $w\left(u_{i}\right)$ of generators $u_{1}, u_{2}, u_{3}, \ldots$ of the algebra $A$ and weights $w\left(L_{i}\right), w(D)$ for the operators $D, L_{1}, L_{2}, L_{3}, \ldots$, which form a basis of the Lie algebra $\mathcal{L}$

$$
w\left(u_{i}\right)=-i, \quad w(D)=-1, \quad w\left(L_{i}\right)=i, \quad \text { where } \quad i \in \mathbb{N} .
$$

A polyonmial Lie algebra $\left(\mathbb{C}\left[u_{1}, u_{2}, \ldots\right], \mathcal{L}\right)$ of a finite rank is defined by means of a countable basis $D, L_{1}, L_{2}, L_{3}, \ldots$ of the Lie algebra $\mathcal{L}$, i.e., by the basis of the left module $\mathcal{L}$ over the algebra of polynomials $A=\mathbb{C}\left[u_{1}, u_{2}, u_{3}, \ldots\right]$.

1) The basis elements $D, L_{1}, L_{2}, L_{3}, \ldots$ should satisfy commutations relations (3.1) and (3.3).

2) The action of the algebra $\mathcal{L}$ on the algebra of polynomials $\mathbb{C}\left[u_{1}, u_{2}, \ldots\right]$ is defined by the action of basis operators on the variables $u_{i}$

$$
D\left(u_{i}\right)=u_{i+1}, \quad L_{1}\left(u_{i}\right)=B_{i-1}\left(u_{1}, \ldots, u_{i-1}\right), \quad L_{2}\left(u_{i}\right)=B_{i-1}\left(-u_{1}, \ldots,-u_{i-1}\right), \quad i \geqslant 1 .
$$

3) The action of basis operators $L_{k}$ for $k \geqslant 3$ is defined by induction beginning with the action of the generators $L_{1}, L_{2}$

$$
L_{3}\left(u_{i}\right)=L_{1} L_{2}\left(u_{i}\right)-L_{2} L_{1}\left(u_{i}\right)=L_{1}\left(B_{i}\left(u_{1}, u_{2}, \ldots, u_{i-1}\right)-L_{2} B_{i}\left(-u_{1},-u_{2}, \ldots,-u_{i-1}\right) .\right.
$$

The commutators $\left[D, L_{k}\right]$ for $k \geqslant 3$ are defined in the same way:

$$
\left[D, L_{3}\right]=\left[D,\left[L_{1}, L_{2}\right]\right]=\left[\left[D, L_{1}\right], L_{2}\right]+\left[L_{1}\left[D, L_{2}\right]\right]=L_{1}-L_{2} .
$$

Analysis of other examples in applications shows that the characteristic Lie algebras can have an additional structure of the Lie-Rinehart algebra, which can significantly simplify search for higher symmetries of hyperbolic systems. This direction is now being actively developed [30]. 
The concept of characteristic algebra can be generalized to the case of semi-discrete and completely discrete hyperbolic equations [20], [22]. If the equation is not symmetric with respect to two independent variables (and in the semi-discrete case this is always like this), then it is necessary to define two characteristic algebras along each of the characteristic directions. We do not provide here the general theory from papers [20], [22], but we only confine ourselves to considering one instructive example and give all the definitions necessary for it.

We consider a scalar equation of form

$$
u_{n+1, x}=f\left(u_{n}, u_{n+1}, u_{n, x}\right),
$$

where $u$ is a function of two independent variables, a continuous variable $x$ and a discrete $n$, the dependence on which is traditionally indicated as a subscript. An analytic function $I=I\left(u_{n, x}, u_{n}, u_{n+1}, u_{n+2}, \ldots\right)$ is called an $x$-integral of equation (3.4) if its total derivative in $x$ by virtue of the equation vanishes: $D(I)=0$, where

$$
D=u_{n, x x} \frac{\partial}{\partial u_{n, x}}+u_{n, x} \frac{\partial}{\partial u_{n}}+f \frac{\partial}{\partial u_{n+1}}+(T f) \frac{\partial}{\partial u_{n+2}}+\left(T^{2} f\right) \frac{\partial}{\partial u_{n+3}} \ldots,
$$

and $T$ is the operator of the shift along the discrete variable: $T f=f\left(u_{n+1}, u_{n+2}, u_{n+1, x}\right)$. It is easy to see that if the function $I$ is an integral of equation (3.4), then it is independent of $u_{n, x}$ since otherwise by applying the operator $D$ we get a term $u_{n, x x}$ which does not cancel out. Hence, each $x$-integral should satisfy the condition $X_{0}(I)=0$, where $X_{0}=\frac{\partial}{\partial u_{n, x}}$.

The Lie algebra $\chi_{x}$ generated by the operators $D$ and $X_{0}$ is called an $x$-characteristic algebra of equation (3.4). It follows from the above that the function $I$ is an $x$-integral of equation (3.4) if and only if it annihilates the characteristic algebra $\chi_{x}$. By analogy with ideas from [1], [2], it is natural to assume that equation (3.4) admits nontrivial $x$-integrals if and only if its characteristic algebra is finite-dimensional. However, as the following example shows, this statement is not true in such form.

Example 3.1. We consider a semi-discrete analogue of the Liouville equation

$$
u_{n+1, x}-u_{n, x}=e^{u_{n+1}}+e^{u_{n}} .
$$

This equation was studied in detail in paper [38], where it was shown that its properties are completely analogous to those of the continuous Liouville equation $u_{x y}=e^{u}$. For example, it possesses an $x$-integral

$$
I=\left(1+e^{u_{n+1}-u_{n+2}}\right)\left(1+e^{u_{n+1}-u_{n}}\right) .
$$

Let us study the characteristic algebra $\chi_{x}$ of this equation. It is easy to confirm that in this case for each $k \in \mathbb{N}$ the identity

$$
T^{k} f=u_{n, x}+e^{u_{n}}+2\left(e^{u_{n+1}}+e^{u_{n+2}}+\cdots+e^{u_{n+k}}\right)+e^{u_{n+k+1}}
$$

holds. This is why the operator $D$ can be represented as $D=X_{0}+u_{n, x} Y+W$, where

$$
Y=\sum_{i=0}^{+\infty} \frac{\partial}{\partial u_{n+i}}, \quad W=\sum_{i=1}^{+\infty}\left(e^{u_{n}}+2 \sum_{j=1}^{i-1} e^{u_{n+j}}+e^{u_{n+i}}\right) \frac{\partial}{\partial u_{n+i}} .
$$

Considering the commutation relations, it is easy to see that

$$
\left[X_{0}, D\right]=Y, \quad[Y, D]=W,
$$

and this implies that $Y, W \in \chi_{x}$. Since $D \in \chi_{x}$, then $u_{n, x} Y=D-X_{0}-W \in \chi_{x}$. We have:

$$
\begin{aligned}
{\left[u_{n, x} Y, W\right] } & =u_{n, x} W \in \chi_{x}, \\
{\left[u_{n, x} Y, u_{n, x} W\right] } & =\left(u_{n, x}\right)^{2} W \in \chi_{x}, \\
{\left[u_{n, x} Y,\left(u_{n, x}\right)^{2} W\right] } & =\left(u_{n, x}\right)^{3} W \in \chi_{x},
\end{aligned}
$$


and so forth. It is easy to see that in this case the characteristic algebra $\chi_{x}$ is infinitedimensional, as a Lie algebra over the field of constants. Nevertheless, it is a finite-dimensional module over the ring of polynomials in the variable $u_{n, x}$ and is generated by the fields $X_{0}, Y$ and $W$ as the Lie-Rinehart algebra.

Characteristic algebras can be defined and effectively employed for studying arbitrary systems of hyperbolic equations. The considered example shows that when passing from exponential systems to the general case (both in the discrete and, generally speaking, in the continuous case), the characteristic algebra should be defined not as a Lie algebra, but as a Lie-Rinehart algebra, and its dimension should be considered in this sense. That is, it is the concept of a Lie-Reinhart algebra that gives a suitable algebraic construction in this case.

\section{GRowth OF CHARACTERISTIC LiE ALGEBRAS}

The emergence and development of the theory of characteristic algebras in its application to the theory of symmetries of hyperbolic systems occurred a little later after the appearance of the first works of Kac and Moody, which laid the foundations of the theory of affine algebras, contragradient Lie algebras, as they were called.

In the end of $60 \mathrm{~s}$, Victor Kac started to study simple $\mathbb{Z}$-graded Lie algebras $\mathfrak{g}=\oplus_{k \in \mathbb{Z}} \mathfrak{g}_{k}$ obeying the following condition for the dimensions of homogeneous components

$$
\operatorname{dim} \mathfrak{g}_{k} \leqslant P(|k|), \quad k \in \mathbb{Z},
$$

where $P(t)$ is some polynomial with non-negative integer coefficients. Kac called such infinite dimensional Lie algebras as Lie algebras of finite growth.

Kac proved [39] that an infinite-dimensional simple $\mathbb{Z}$-graded Lie algebra $\mathfrak{g}$ of finite growth satisfying some two technical conditions is isomorphic to one (and only one) Lie algebra from the following list (see [40] to clarify definitions and notation)

- Centerless affine algebras: six infinite series and seven exceptional algebras

$A_{n}^{(1)}, \quad B_{n}^{(1)}, \quad C_{n}^{(1)}, \quad D_{n}^{(1)}, \quad A_{n}^{(2)}, \quad D_{n}^{(2)}, \quad E_{6}^{(2)}, \quad D_{4}^{(3)}, \quad E_{6}^{(1)}, \quad E_{7}^{(1)}, \quad E_{8}^{(1)}, \quad F_{4}^{(1)}, \quad G_{2}^{(1)} ;$

- Lie algebras of Cartan type $W_{n}, S_{n}, K_{n}, H_{n}$.

In addition to his theorem, Kac conjectured that omitting the additional technical conditions from his theorem would add to this list only one simple $\mathbb{Z}$-graded Lie algebra, the Witt algebra $W$. This conjecture was proved by Kac by Mathieu in 1990 [40].

The appearance of the notion growth of a Lie algebra in Kac's work was also not accidental since the theory of the growth of groups and algebras appeared and became very popular precisely in those years [41]: the end of the 60s and the beginning of the 70s; the concept of the growth of a group appeared a bit earlier. The notion finite growth from paper [39] still did not take root in the algebraic literature, usually now one says about polynomial growth.

Let us give a modern definition of the growth function of a Lie algebra, see for details [41. Suppose that an infinite-dimensional Lie algebra $\mathfrak{g}$ is generated by a finite-dimensional subspace $V_{1}(\mathfrak{g})$. We denote by $V_{n}(\mathfrak{g})$ the subspace $V_{1}(\mathfrak{g})$ spanned over higher commutators of length at most $n \geqslant 2$ with an arbitrary arrangement of parentheses. A chain of infinite-dimensional subspaces appears:

$$
V_{1}(\mathfrak{g}) \subset V_{2}(\mathfrak{g}) \subset \cdots \subset V_{n}(\mathfrak{g}) \subset \ldots, \quad \cup_{i=1}^{+\infty} V_{i}(\mathfrak{g})=\mathfrak{g} .
$$

We define the growth function $F_{\mathfrak{g}}(n)=\operatorname{dim} V_{n}(\mathfrak{g})$. However, such definition is not invariant since it depends on the choice of the generating set $V_{1}$.

For instance, if we choose another generating set $\tilde{V}_{1}(\mathfrak{g})$ such that $\tilde{V}_{1}(\mathfrak{g}) \subset V_{m}(\mathfrak{g})$ for some natural $m$, then we obtain the following estimate for two growth functions

$$
\tilde{F}_{\mathfrak{g}}(n)=\operatorname{dim} \tilde{V}_{n}(\mathfrak{g}) \leqslant \operatorname{dim} V_{m n}(\mathfrak{g})=F_{\mathfrak{g}}(m n) .
$$


A growth of Lie algebra $\mathfrak{g}$ is an equivalence class of its growth functions [41]. Growth functions $f, g: \mathbb{N} \rightarrow \mathbb{N}$ are called equivalent if there exists constants $c, m, \tilde{c}, \tilde{m} \in \mathbb{N}$ such that

$$
f(n) \leqslant c g(m n), \quad g(n) \leqslant \tilde{c} f(\tilde{m} n)
$$

for almost each $n \in \mathbb{N}$.

One chooses three growth types of Lie algebras: 1) polynomial; 2) exponential (this is the growth of a free Lie algebra of finitely many generators); 3) intermediate or sub-exponential growth.

It is convenient to characterize the polynomial growth type by means of another invariant called Gelfand-Kirillov dimension [42]

$$
\operatorname{GKdim} \mathfrak{g}=\limsup _{n \rightarrow+\infty} \frac{\log \operatorname{dim} V_{n}(\mathfrak{g})}{\log n} .
$$

The finiteness of the Gelfand-Kirillov dimension GKdim $\mathfrak{g}$ means that there exists a polynomial $P(x)$ such that $\operatorname{dim} V_{n}(\mathfrak{g})<P(n)$. If the Lie algebra $\mathfrak{g}$ is finite-dimensional, then its GelfandKirillov dimension vanishes: GKdim $\mathfrak{g}=0$. Informally, we can also say the following: if the Gelfand-Kirillov dimension of the Lie algebra $\mathfrak{g}$ is equal to some real number $\alpha$, then the growth function of the Lie algebra $\mathfrak{g}$ grows at the rate of the power function $C n^{\alpha}$, where $C$ is some positive constant.

While studying the growth of characteristic Lie algebras, one should have in mind a number of additional circumstances. The first of them is reflected in the next lemma, which characterizes, in particular, the characteristic Lie algebras of exponential systems corresponding to the Cartan matrices.

Lemma $4.1([34])$. Let $\tilde{\mathfrak{g}}$ be an infinite-dimensional Lie algebra generated by a finitedimensional subspace

$$
V_{1}(\tilde{\mathfrak{g}})=\mathfrak{g}_{0} \oplus \mathfrak{g}_{1},
$$

where $\mathfrak{g}_{0}$ is a commutative Lie subalgebra in $\tilde{\mathfrak{g}}$, and the subspace $\mathfrak{g}_{1}$ of dimension $q$ is invariant under the action of $\mathfrak{g}_{0}$ to $\tilde{\mathfrak{g}}$. We suppose also that the $\mathfrak{g}_{0}$-module $\mathfrak{g}_{1}$ is diagonalizable and its corresponding weights $\alpha_{1}, \ldots, \alpha_{q} \in \mathfrak{g}_{0}^{*}$ are non-trivial. We consider the subalgebra $\mathfrak{g}$ in $\mathfrak{g}$ generated by the subspace $\mathfrak{g}_{1}$. Then the growth functions $F_{\mathfrak{g}}(n)$ and $F_{\mathfrak{g}}(n)$ differ one from the other by a constant

$$
F_{\tilde{\mathfrak{g}}}(n)=F_{\mathfrak{g}}(n)+\operatorname{dim} \mathfrak{g}_{0} .
$$

Corollary 4.1. Lie algebras $\mathfrak{g}$ and $\tilde{\mathfrak{g}}$ have the same Gelfand-Kirillov dimensions.

In the already discussed example on the characteristic Lie algebra $\tilde{\mathfrak{g}}=\chi(\sinh (u))$ of sineGordon equation, the commutative Lie algebra $\mathfrak{g}_{0}=\left\langle X_{0}\right\rangle$ is one-dimensional and the twodimensional space $\mathfrak{g}_{1}=\left\langle X_{1}, X_{2}\right\rangle$ was defined as the linear span

$$
\mathfrak{g}_{1}=\left\langle X(\sinh (u)),\left[X_{0}, X(\sinh (u))\right]\right\rangle .
$$

The general conclusion is as follows: if the characteristic Lie algebra $\tilde{\mathfrak{g}}$ satisfies the assumptions of Lemma 4.1, then we can study the growth of its commutant $\mathfrak{g}=[\tilde{\mathfrak{g}}, \tilde{\mathfrak{g}}]$ instead of the growth of the entire Lie algebra $\tilde{\mathfrak{g}}$.

The second property describing the characteristic Lie algebras $\tilde{\mathfrak{g}}$ of exponential systems corresponding to Cartan matrices is the natural positive grading of their commutants $\mathfrak{g}=[\tilde{\mathfrak{g}}, \tilde{\mathfrak{g}}]$.

Definition 4.1. $\mathbb{N}$-graded Lie algebra $\mathfrak{g}=\oplus_{i=1}^{+\infty} \mathfrak{g}_{i}$ is called naturally graded if

$$
\left[\mathfrak{g}_{1}, \mathfrak{g}_{k}\right]=\mathfrak{g}_{k+1}, \quad \text { where } \quad k \geqslant 1
$$


For a naturally graded Lie algebra $\mathfrak{g}=\bigoplus_{i=1}^{+\infty} \mathfrak{g}_{i}$ we can define one specific growth function $F_{\mathfrak{g}}^{g r}(n)$ [34] choosing a homogeneous component $\mathfrak{g}_{1}$ as the generating subspace $V_{1}(\mathfrak{g})$. The following properties are obvious:

$$
F_{\mathfrak{g}}^{g r}(n)=\operatorname{dim} V_{n}(\mathfrak{g})=\sum_{i=1}^{n} \operatorname{dim} \mathfrak{g}_{i}=\operatorname{dim}\left(\mathfrak{g} / \mathfrak{g}^{n+1}\right),
$$

where $\mathfrak{g}^{n+1}$ stands for $(n+1)$ th ideal of the lower central series of Lie algebra $\mathfrak{g}$.

Definition 4.2. $\mathbb{N}$-graded Lie algebra $\mathfrak{g}=\oplus_{i=1}^{+\infty} \mathfrak{g}_{i}$ is called Lie algebra of a bounded width if the dimensions of all its homogeneous components are uniformly bounded by some constant $C$

$$
\operatorname{dim} \mathfrak{g}_{k} \leqslant C, \text { where } k \geqslant 1 \text {. }
$$

The maximal dimension among the dimensions $\operatorname{dim} \mathfrak{g}_{k}$ of homogeneous components of a Lie algebra of a finite width is called its width $d(\mathfrak{g})$.

For an arbitrary naturally graded Lie algebra $\mathfrak{g}=\oplus_{i=1}^{+\infty} \mathfrak{g}_{i}$ of width $d(\mathfrak{g})$ the function $F_{\mathfrak{g}}^{g r}(n)$ grows not faster than $d(\mathfrak{g}) n$ :

$$
F_{\mathfrak{g}}^{g r}(n) \leqslant d(\mathfrak{g}) n .
$$

Therefore, the Gelfand-Kirillov dimension GKdim $\mathfrak{g}$ of an arbitrary Lie algebra of finite width is equal to one: $\operatorname{GKdim} \mathfrak{g}=1$.

It was shown in paper [34] that the characteristic Lie algebras of the sine-Gordon and Tzizeica equations grow with the average linear rates $\frac{3}{2}$ and $\frac{4}{3}$, respectively. We shall show in Section 6 that the characteristic algebras of systems corresponding to degenerate Cartan matrices of rank 2 also have linear growth.

In a number of works, in particular in monograph [31, it was conjectured that the integrability of exponential hyperbolic nonlinear systems is determined by the growth of the corresponding characteristic Lie algebra. It seems to us that both the integrability and the slow growth of characteristic Lie algebras are indirect manifestations of the properties of the Cartan matrices. In any case, one should not rely too much on the restrictions that have arisen only due to slow growth. Note also that the $\mathbb{Z}$-graded Lie algebras from the Kac list are simple and this is a very strong restriction. If the characteristic Lie algebras have a grading, then it is nonnegative, and this means that in principle they cannot be simple. A number of additional restrictions is to be added to the conditions for slow growth. All this can be considered only as a plan for future research.

\section{COMBinatorics OF Liouville EQUATION}

This section is devoted to the properties of integrals and symmetries of the Liouville equation from the point of view of formal algebra and combinatorics. We consider the Liouville equation $u_{x y}=f(u)=e^{u}$. By straightforward calculations one confirm easily that the polynomial

$$
q_{2}=2 u_{2}-u_{1}^{2}=2 u_{x x}-u_{x}^{2}
$$

is a $y$-integral of the Liouville equation.

We define a polynomial differential operator $X$ by the formula $X_{e^{u}}=e^{u} X$, that is,

$$
X=\frac{\partial}{\partial u_{1}}+u_{1} \frac{\partial}{\partial u_{2}}+\left(u_{1}^{2}+u_{2}\right) \frac{\partial}{\partial u_{2}}+\ldots
$$


The coefficients of this operator are known in combinatorics as the complete Bell polynomials $B_{k}$

$$
X=\sum_{k=1}^{\infty} B_{k-1}\left(u_{1}, \ldots, u_{k-1}\right) \frac{\partial}{\partial u_{k}} .
$$

In book by Riordan [43] one can find many interesting combinatorial properties of Bell polynomials, they also have many applications.

A generating function for the Bell polynomial $B_{n}\left(u_{1}, u_{2}, \ldots, u_{n}\right)$ is

$$
\exp \left(\sum_{i=1}^{+\infty} u_{i} \frac{t^{i}}{i !}\right)=\sum_{n=0}^{+\infty} B_{n}\left(u_{1}, \ldots, u_{n}\right) \frac{t^{n}}{n !}
$$

In [43], also the following recurrent formula was given for the Bell polynomials $B_{n}\left(u_{1}, \ldots, u_{n}\right)$ :

$$
\left(D+u_{1}\right) B_{k}=B_{k+1}, \quad k \geqslant 0 .
$$

Corollary 5.1. The identity holds:

$$
B_{n}\left(u_{1}, \ldots, u_{n}\right)=\left(D+u_{1}\right)^{n}(1) .
$$

It is convenient to write recurrently the Bell polynomials by means of formula (5.1):

$$
\begin{aligned}
B_{1}\left(u_{1}\right) & =\left(D+u_{1}\right)(1)=u_{1}, \\
B_{2}\left(u_{1}, u_{2}\right) & =\left(D+u_{1}\right)\left(u_{1}\right)=u_{2}+u_{1}^{2}, \\
B_{3}\left(u_{1}, u_{2}, u_{3}\right) & =\left(D+u_{1}\right)\left(u_{2}+u_{1}^{2}\right)=u_{3}+3 u_{1} u_{2}+u_{1}^{3}, \\
B_{4}\left(u_{1}, u_{2}, u_{3}, u_{4}\right) & =\left(D+u_{1}\right)\left(B_{3}\left(u_{1}, u_{2}, u_{3}\right)\right)=u_{1}^{4}+6 u_{1}^{2} u_{2}+4 u_{1} u_{3}+3 u_{2}^{2}+u_{4} .
\end{aligned}
$$

The complete Bell polynomials can be recurrently defined in one more way:

$$
B_{0}=1, \quad B_{n+1}\left(u_{1}, u_{2}, \ldots, u_{n+1}\right)=\sum_{i=0}^{n}\left(\begin{array}{c}
n \\
i
\end{array}\right) B_{n-i}\left(u_{1}, u_{2}, \ldots, u_{n-i}\right) u_{i+1} .
$$

We define the grading in the algebra of polynomials $A=\mathbb{K}\left[u_{1}, u_{2}, u_{3}, \ldots\right]$ on its generators

$$
w\left(u_{k}\right)=k, \quad k=1,2,3, \ldots,
$$

continuing then it by the multiplicativity for all polynomials. For instance, the polynomial $q_{2}=2 u_{2}-u_{1}^{2}$ is homogeneous of weight 2 . We note that this grading differs by sign from the grading of the algebra $A=\mathbb{K}\left[u_{1}, u_{2}, u_{3}, \ldots\right]$ introduced in Section 3 .

Thus, the algebra $A$ is positively graded

$$
A=\oplus_{n=1}^{+\infty} A_{n}, \quad A_{n}=\{P \in A, w(P)=n\} .
$$

The operator $X$ decreases the weight $w(P)$ of each homogeneous polynomial $P \in A_{n}$ by one, while the operator $D$ and the operator of multiplication by $u_{1}$ do vice versa: these operators increase the weight of each homogeneous polynomial exactly by one

$$
X: A_{n} \rightarrow A_{n-1}, \quad D: A_{n} \rightarrow A_{n+1}, \quad u_{1}: A_{n} \rightarrow A_{n+1}, \quad n \geqslant 1 .
$$

We define an increasing filtration $\left\{A^{m}, m \geqslant 0\right\}$ of the algebra $A$

$A^{0}=\langle 1\rangle \subset A^{1}=\left\langle 1, u_{1}\right\rangle \subset A^{2}=\left\langle 1, u_{1}, u_{1}^{2}, u_{2}\right\rangle \subset \cdots \subset A^{m}=\{P \in A, w(P) \leqslant m\} \subset A^{m+1} \subset \ldots$, that is, we define a filtrating subspace $A^{m}$ as the linear span of homogeneous polynomials $P$ with weights $w(P)$ at most $m$.

Proposition 5.1. The operator $X$ restricted to an arbitrary finite-dimensional subspace $A^{m}$ becomes nilpotent: $\left.X\right|_{A^{m}} ^{m+1}=0$. 
Since $X$ is a differentiation of the algebra $A$, its kernel $\operatorname{Ker} X$ is a subalgebra of $A$. Moreover, the subalgebra $\operatorname{Ker} X$ is $D$-invariant due to the following commutation relations

$$
[D, X]=D X-X D=-u_{1} X \quad \Leftrightarrow \quad\left(D+u_{1}\right) X=X D .
$$

In what follows we shall make use of the following generalization of commutation relation (5.3)

$$
X\left(D+k u_{1}\right)=\left(D+(k+1) u_{1}\right) X+k \mathrm{Id}, \quad k \in \mathbb{Z} .
$$

We consider homogeneous $y$-integrals of weights $3,4, \ldots, k, \ldots$, which are defined recurrently using the operator $D$ and the very first element of the kernel $q_{2}=2 u_{2}-u_{1}^{2}$ (other than a constant):

$$
q_{3}=D\left(q_{2}\right)=2 u_{1} u_{2}-2 u_{3}, \quad q_{4}=D^{2}\left(q_{2}\right)=2 u_{2}^{2}+2 u_{1} u_{3}-2 u_{4}, \quad \ldots, \quad q_{k}=D^{k-2}\left(q_{2}\right), \quad \ldots
$$

We can treat the polynomials $q_{k}$ as a polynomial deformation of the variables $2 u_{k}$

$$
q_{k}=2 u_{k}+Q_{k}\left(u_{1}, \ldots, u_{k-1}\right),
$$

where the quadratic polynomials $Q_{k}\left(u_{1}, \ldots, u_{k-1}\right)$ depend on the variables $u_{j}$ with the indices $j$ strictly less than $k$. Hence, the polynomials of form $q_{2}^{k_{2}} q_{3}^{k_{3}} \ldots q_{m}^{k_{m}}$, where $k_{i} \in \mathbb{Z}_{\geqslant 0}, m \geqslant 1$, form an infinite set of linearly independent polynomials.

We arrive at the following well-known theorem.

Theorem 5.1 (Shabat, Zhiber, 1979). The subalgebra Ker $X$ is isomoprhic to a polynomial algebra $\mathbb{K}\left[q_{2}, q_{3}, \ldots, q_{k}, \ldots\right]$, where $q_{k}=D^{k-2}\left(q_{2}\right), k \geqslant 2$.

Concerning the proof of this algebraic theorem, at present, only the inclusion of subalgebras

$$
\mathbb{K}\left[q_{2}, q_{3}, q_{4}, \ldots\right] \subset \operatorname{Ker} X
$$

is proved. A bit later in this section we shall prove that this inclusion is in fact the identity.

By means of the operator $X$ we define one more increasing filtration of the algebra $A$

$$
\tilde{A}^{0}=\{0\} \subset \tilde{A}^{1} \subset \tilde{A}^{2} \subset \cdots \subset \tilde{A}^{m}=\left\{P \in A, X^{m} P=0\right\} \subset \tilde{A}^{m+1} \subset \ldots,
$$

that is, in the case the filtrating subspace $\tilde{A}^{m}$ is defined as the kernel of the operator $X^{m}$. We note that the subspaces $\tilde{A}^{m}$ are no longer finite-dimensional. In addition, there exists an obvious relation between two filtrations due to Proposition 5.1

$$
A^{m} \subset \tilde{A}^{m+1}, \quad m \geqslant 0 .
$$

We also note that $\tilde{A}^{1}=\operatorname{Ker} X$.

For all natural $m$, the inclusions hold:

$$
X \tilde{A}^{m} \subset \tilde{A}^{m-1}, \quad u_{1} \tilde{A}^{m} \subset \tilde{A}^{m+1}, \quad D \tilde{A}^{m} \subset \tilde{A}^{m+1} .
$$

The first inclusion is implied by the definition of $\tilde{A}^{m}$, while to prove the other, it is sufficient to note that the relations hold:

$$
X^{m+1}\left(u_{1} F\right)=(m+1) X^{m} F+u_{1} X^{m+1} F, \text { where } m \geqslant 0,
$$

which can be proved by a simple induction in $m$ starting from the obvious identity

$$
X\left(u_{1} F\right)=F+u_{1} X F .
$$

The latter inclusion is implied by the following relation involving the operator $D$ :

$$
X^{m+1}(D F)=\left(D+(m+1) u_{1}\right) X^{m+1} F+\frac{m(m+1)}{2} X^{m} F, \quad m \geqslant 1 .
$$


It is also proved by induction in the exponent $m$ starting from $(5.3)$ :

$$
\begin{aligned}
X^{m+1} D F & =X\left(D+m u_{1}\right) X^{m} F+X\left(\frac{(m-1) m}{2} X^{m-1} F\right) \\
& =\left(D+(m+1) u_{1}\right) X^{m+1} F+\left(\frac{(m-1) m}{2}+m\right) X^{m} F .
\end{aligned}
$$

As an implication of (5.7), (5.8) we obtain the following formula:

$$
X^{m+1}\left(D+k u_{1}\right) F=\left(D+(m+k+1) u_{1}\right) X^{m+1} F+\frac{(m+1)(m+2 k)}{2} X^{m} F .
$$

Remark 5.1. The polynomials $P$ in the kernel of the operator Ker $X$ are the eigenvectors of the operator $X D$ associated with the eigenvalue $\lambda=0$ :

$$
X D(P)=\left(D+u_{1}\right) X P=0 .
$$

Lemma 5.1. Let $F$ be an arbitrary polynomial in the algebra $A$, then the series

$$
\pi(F)=F-u_{1} X F+\frac{u_{1}^{2}}{2 !} X^{2} F-\frac{u_{1}^{3}}{3 !} X^{3} F+\cdots+(-1)^{k} \frac{u_{1}^{k}}{k !} X^{k} F+\ldots
$$

contains finitely many non-zero terms and the polynomial $\pi F$ annihilates the operator $X$.

The proof of the lemma is the straightforward calculation

$$
X \pi(F)=X F-X\left(u_{1}\right) X F-u_{1} X^{2} F+\frac{X\left(u_{1}^{2}\right)}{2 !} X^{2} F-\ldots
$$

Since

$$
X\left(u_{1}\right)=1, \quad X\left(u_{1}^{2}\right)=2 u_{1}, \quad X\left(u_{1}^{k}\right)=k u_{1}^{k-1},
$$

then in the case when $F \in A^{m}$ for some natural $m$, we have:

$$
X \tilde{F}=\frac{X^{m}}{m !} F=0 .
$$

In particular, it follows from the proof that for the polynomial $F \in \tilde{A}^{m+1}$ we have

$$
\pi(F)=F-u_{1} X F+\frac{u_{1}^{2}}{2 !} X^{2} F-\frac{u_{1}^{3}}{3 !} X^{3} F+\cdots+(-1)^{m} \frac{u_{1}^{m-1}}{(m-1) !} X^{m} F .
$$

In particular, for $F \in \operatorname{Ker} X^{2}$ we have $F-u_{1} X F \in \operatorname{Ker} X$.

Remark 5.2. The mapping $\pi: A \rightarrow A$ defined by formula (5.10) is a projector on the subalgebra $\operatorname{Ker} X: \pi^{2}=\pi$.

It is easy to find the kernel of this projector:

$$
\text { Ker } \pi=u_{1} A=\left\{u_{1} F, F \in A\right\} .
$$

Indeed, we employ formula $(5.7)$ to calculate $\pi\left(u_{1} F\right)$ for an arbitrary $F \in A$

$$
\begin{aligned}
\pi\left(u_{1} F\right) & =u_{1} F-u_{1} X\left(u_{1} F\right)+\frac{u_{1}^{2}}{2 !} X^{2}\left(u_{1} F\right)-\frac{u_{1}^{3}}{3 !} X^{3}\left(u_{1} F\right)+\ldots \\
& =u_{1} F-u_{1} F-u_{1}^{2} X F+\frac{u_{1}^{2}}{2 !}\left(2 X F+u_{1} X^{2} F\right)-\frac{u_{1}^{3}}{3 !}\left(3 X^{2} F+u_{1} X^{3} F\right)+\cdots=0 .
\end{aligned}
$$

As an implication, we obtain the formula for the dimension of the kernel of the operator $X$ restricted to the subspace $A^{n}$ of elements of weight $n$ :

$$
\left.\operatorname{dim} \operatorname{Ker} \pi\right|_{A^{n}}=\operatorname{dim} u_{1} A^{n-1}=p(n-1),
$$

according to (5.11). This gives rise to the formula

$$
\left.\operatorname{dim} \operatorname{Ker} X\right|_{A^{n}}=\left.\operatorname{dim} \operatorname{Im} \pi\right|_{A^{n}}=\operatorname{dim} A^{n}-\left.\operatorname{dim} \operatorname{Ker} \pi\right|_{A^{n}}=p(n)-p(n-1),
$$


where $p(n)$ denotes the number of partitions of a number $n$, while the difference $p(n)-p(n-1)$ is obviously equal to the number of partitions of $n$ into the sum of terms strictly greater than 1: the number of partitions of $n$ containing the unit is obtained from some partition of $n-1$, to which we add one unit.

Corollary 5.2. Inclusion (5.5) in Zhiber-Shabat theorem 5.1 is an isomorphism.

We have also proved that the operator $\left.X\right|_{A^{n}}: A^{n} \rightarrow A^{n-1}$ is a mapping of a maximal rank: $\left.\operatorname{dim} \operatorname{Im} X\right|_{A^{n}}=\operatorname{dim} A^{n-1}=p(n-1)$.

Let us recall the defining equation of higher symmetries of the Klein-Gordon equation (the equation of the formal Lie-Bäcklund group of higher symmetries)

$$
F_{x y}=f^{\prime}(u) F, \quad u_{\tau}=F\left(u, u_{x}, u_{x x}, \ldots\right),
$$

which can be reduced to the following algebraic form

$$
\left(D+u_{1}\right) X F=X D F=F .
$$

This means that the symmetry $F=F\left(u_{1}, u_{2}, \ldots\right)$ is an eigenvector of the operator $X D$ associated with the eigenvalue $\lambda=1$.

Lemma 5.2. For all integer $m$, the operator $D+m u_{1}$ has a zero kernel:

$$
\operatorname{Ker}\left(D+m u_{1}\right)=\{0\} .
$$

We use the standard lexicographic ordering in the algebra $A$ and select the highest monomial $\gamma u_{1}^{k_{1}} \ldots u_{n}^{k_{n}}$ in the expansion of an element in the kernel $F \in \operatorname{Ker}\left(D+m u_{1}\right)$. This means, in particular, that $u_{n}$ is the highest variable among those participating in the expansion of the polynomial $F$ :

$$
F=\gamma u_{1}^{k_{1}} \ldots u_{n}^{k_{n}}+\ldots, \quad \gamma \in \mathbb{C} .
$$

The dots stands for the sums of monomials of variables $u_{1}, \ldots, u_{n}$, in which the variable $u_{n}$ can be involved with multiplicities strictly less than $k_{n}$. We apply the operator $\left(D+m u_{1}\right)$ to $F$ :

$$
D F+u_{1} F=\gamma k_{n} u_{1}^{k_{1}} \ldots u_{n}^{k_{n}-1} u_{n+1}+\ldots,
$$

that is, the monomial $\gamma k_{n} u_{1}^{k_{1}} \ldots u_{n}^{k_{n}-1} u_{n+1}$ is the highest in the expansion of $\left(D+m u_{1}\right) F$ since the multiplicity of the variable $u_{n}$ in the monomial, where $u_{n+1}$ is present, is maximal. Hence, $\gamma=0$ and by similar reasoning we obtain that $F=0$.

Proposition 5.2. Let $F$ be a symmetry, that is, it is an eigenvector of the operator XD with $\lambda=1$. Then $X^{2}(F)=0$.

By the definition of the symmetry $F=X D F$ and commutation relations (5.4) for $k=1$ we obtain the following chain of identities

$$
X F=X^{2} D F=X\left(D+u_{1}\right) X F=\left(\left(D+2 u_{1}\right) X+I d\right) X F=\left(D+2 u_{1}\right) X^{2} F+X F .
$$

This implies that $\left(D+2 u_{1}\right) X^{2} F=0$ and applying Lemma 5.2, we arrive at the statement of the proposition.

Theorem 5.2 (Zhiber, Shabat, 1979, [32]). An arbitrary $x$-symmetry $F$, an eigenvector of the operator $X D$ with $\lambda=1$, can be written as

$$
F=\left(D+u_{1}\right) Q, \quad Q \in \operatorname{Ker} X=\mathbb{K}\left[q_{2}, q_{3}, \ldots\right] .
$$

We reproduce here in a more rigorous way the proof from [32]. In one direction: let $X Q=0$, then

$$
\begin{aligned}
\left(D+u_{1}\right) X\left(D+u_{1}\right) Q & =\left(D+u_{1}\right)\left(\left(D+2 u_{1}\right) X+I d\right) Q \\
& =\left(D+u_{1}\right)\left(D+2 u_{1}\right) X Q+\left(D+u_{1}\right) Q=\left(D+u_{1}\right) Q .
\end{aligned}
$$


The proof in the opposite direction is implied by the definition of the symmetry $\left(D+u_{1}\right) X F=$ $F$. We introduce an element $Q=X F$. It follows from Proposition 5.2 that $F=\left(D+u_{1}\right) Q$ and, in addition, $X Q=X^{2} F=0$.

We denote the eigenspaces of the operator $X D$ associated with $\lambda=0,1$ respectively by $V_{0}$ and $V_{1}$.

Proposition 5.3. The identity holds:

$$
V_{0} \oplus V_{1}=\operatorname{Ker} X^{2} .
$$

We have proved the inclusion $V_{0} \oplus V_{1} \subset \operatorname{Ker} X^{2}$. We consider a finite-dimensional version by restricting all operators to the subspace $A^{n}$ of polynomials of weight $n$. The subspace $V_{1}(n)=V_{1} \cap A^{n}$ is isomorphic to the subspace $V_{0}(n-1)=V_{0} \cap A^{n-1}$ according to the theorem 5.2 and Lemma 5.2. Hence,

$$
\operatorname{dim}\left(V_{0}(n) \oplus V_{1}(n)\right)=p(n)-p(n-1)+p(n-1)-p(n-2)=p(n)-p(n-2) .
$$

On the other hand, the operator $X: A^{n} \rightarrow A^{n-1}$ is surjective for each $n$, and hence the same is true for the operator $X^{2}: A^{n} \rightarrow A^{n-2}$. Therefore, $\left.\operatorname{dim} \operatorname{Ker} X^{2}\right|_{A^{n}}=p(n)-p(n-2)$.

A natural question arises: "What other eigenvectors are there for the operator $X D$ ?"

Before giving a general answer to this question, let us analyze examples in small dimensions.

1) Weight $n=1$. The operator $X D$ has a single eigenvector $u_{1}$ associated with the eigenvalue $\lambda_{1}=1$.

2) Weight $n=2$. We consider the basis $u_{1}^{2}, u_{2}$. Then the matrix of the operator $X D$ reads as $\left(\begin{array}{ll}2 & 1 \\ 2 & 1\end{array}\right)$. This matrix has two eigenvalues: $\lambda_{0}=0$ and $\lambda_{2}=3$. The corresponding eigenvectors are two polynomials

$$
q_{2}=u_{1}^{2}-2 u_{2}, \quad B_{2}=u_{1}^{2}+u_{2} .
$$

3) In the weight $n=3$ we fix the basis $u_{1}^{3}, u_{1} u_{2}, u_{3}$. In this basis, the matrix of the operator $X D$ is

$$
\left(\begin{array}{lll}
3 & 1 & 1 \\
6 & 3 & 3 \\
0 & 1 & 1
\end{array}\right)
$$

Its eigenvalues are $\lambda_{0}=0, \lambda_{1}=1$ and $\lambda_{3}=6$. The associated eigenvectors are respectively of the form

$$
\begin{array}{ll}
q_{3}=u_{1} u_{2}-2 u_{3}, & \left(\lambda_{0}=0\right), \\
u_{1}^{3}-2 u_{3}, & \left(\lambda_{1}=1\right), \\
2 u_{1}^{2}+5 u_{1} u_{2}+u_{3}, & \left(\lambda_{3}=6\right) .
\end{array}
$$

4) In the weight $n=4$ we choose the basis of the polynomials $u_{1}^{4}, u_{1}^{2} u_{2}, u_{1} u_{3}, u_{2}^{2}, u_{4}$. Now the matrix of the operator $X D$ is of size $5 \times 5$ :

$$
\left(\begin{array}{ccccc}
4 & 1 & 1 & 0 & 1 \\
12 & 5 & 4 & 2 & 6 \\
0 & 2 & 2 & 2 & 4 \\
0 & 2 & 1 & 2 & 3 \\
0 & 0 & 1 & 0 & 1
\end{array}\right)
$$

Its eigenvalues are $\lambda_{0}=0$ (of multiplicity 2), $\lambda_{1}=1, \lambda_{2}=3$ and $\lambda_{4}=10$. Among the eigenvectors of this matrix we have two integrals $q_{2}^{2}$ and $q_{4}\left(\lambda_{0}=0\right)$, one symmetry $(D+$ $\left.u_{1}\right)\left(q_{3}\right)=q_{4}$, and other eigenvectors:

$$
-u_{1}^{4}-2 u_{1}^{2} u_{2}+2 u_{1} u_{3}+u_{2}^{2}+u_{4} \quad \text { and } \quad 6 u_{1}^{4}+26 u_{1}^{2} u_{2}+9 u_{1} u_{3}+8 u_{2}^{2}+u_{4} \text {, }
$$


associated respectively with $\lambda_{2}=3$ and $\lambda_{4}=10$.

We arrive to a theorem, which naturally generalizes Theorem 5.2 .

Theorem 5.3. 1) The operator $\left(D+u_{1}\right) X=X D$ restricted to the subspace, $A_{n}$ of polynomials of weight $n \geqslant 2$ is diagonalizable;

2) Its spectrum is the following set of nonnegative integer numbers:

$$
\lambda_{0}=0, \quad \lambda_{1}=1, \ldots, \quad \lambda_{n-2}=\frac{(n-2)(n-1)}{2}, \quad \lambda_{n}=\frac{n(n+1)}{2} ;
$$

3) The multiplicity of the eigenvalue $\lambda_{k}$ is equal to

$$
p(n-k)-p(n-k-1), \quad k=0,1, \ldots, n-2, n ;
$$

4) An arbitrary eigenvectors $P$ associated with an eigenvalue $\lambda_{k}$, where $k \in\{0,1, \ldots, n-2, n\}$, can be written as

$$
P=\left(D+u_{1}\right)\left(D+2 u_{1}\right) \ldots\left(D+k u_{1}\right) F,
$$

where $F$ denotes some homogeneous polynomial of weight $(n-k)$ in the kernel $\operatorname{Ker} X$.

We begin the proof with Statement 4 .

Let $X F=0$. We apply the operator $X D$ to the polynomial $P=\left(D+u_{1}\right)\left(D+2 u_{1}\right) \ldots(D+$ $\left.n u_{1}\right) F:$

$$
\begin{aligned}
X D\left(D+u_{1}\right)\left(D+2 u_{1}\right) \ldots\left(D+n u_{1}\right) F & =\left(D+u_{1}\right) X\left(D+u_{1}\right)\left(D+2 u_{1}\right) \ldots\left(D+n u_{1}\right) F \\
& =\left(D+u_{1}\right)\left(\left(D+2 u_{1}\right) X+i d\right)\left(D+2 u_{1}\right) \ldots\left(D+n u_{1}\right) F \\
& =\left(D+u_{1}\right)\left(D+2 u_{1}\right) X\left(D+3 u_{1}\right) \ldots\left(D+n u_{1}\right) F+P .
\end{aligned}
$$

Swapping the operators $X$ and $\left(D+k u_{1}\right)$ and taking into account relation (5.4), we finally end up with the relation

$$
X D P=\left(D+u_{1}\right)\left(D+2 u_{1}\right) \ldots\left(D+n u_{1}\right) X F+P+2 P+\cdots+n P=\frac{n(n+1)}{2} P,
$$

where we have employed that $X F=0$.

The next proposition is an elementary implication of lemma 5.2 .

Proposition 5.4. A linear mapping $\varphi_{k}: V_{0}(n-k) \rightarrow V_{k}(n)$ defined by the formula

$$
\varphi_{k}(F)=\left(D+u_{1}\right)\left(D+2 u_{1}\right) \ldots\left(D+k u_{1}\right) F,
$$

is a monomorphism.

It implies immediately a simple bound for the dimensions of eigenspaces $V_{k}(n)$ :

$$
\operatorname{dim} V_{k}(n) \geqslant \operatorname{dim} \operatorname{Im} \varphi_{k}=\operatorname{dim} V_{0}(n-k)=\operatorname{dim} \operatorname{Ker}_{n-k} X=p(n-k)-p(n-k-1),
$$

where $p(n)$ denotes the number of partitions of $n$.

We consider the direct sum $\oplus_{k=0, \neq n-1}^{n} V_{k}(n)$ of eigensubspaces of the operator $\left.X D\right|_{A_{n}}$. According to (5.13), we have the following estimate for its dimension

$$
\operatorname{dim}\left(\oplus_{k=0, \neq n-1}^{n} V_{k}(n)\right) \geqslant \sum_{k=0}^{n-2}(p(n-k)-p(n-k-1))+1=p(n)=\operatorname{dim} A_{n} .
$$

Hence, we conclude that this inequality is identity and the same is true for all inequalities (5.13). This implies Statement 1), that is,

$$
A_{n}=V_{0}(n) \oplus V_{1}(n) \oplus \cdots \oplus V_{n-2}(n) \oplus V_{n}(n),
$$

and Statement 2) on the dimensions of eigenspaces $V_{k}$. The proof of the theorem is complete. 
Corollary 5.3. There is a simple relation between the eigensubspaces of $V_{k}$ of operator $X D$ and the filtering $\tilde{A}^{m}$ of the polynomial algebra $A$

$$
V_{0} \oplus V_{1} \oplus \cdots \oplus V_{k}=\operatorname{Ker} X^{k+1}=\tilde{A}^{k+1} .
$$

We have already proved this statement for $k=0,1$. Here the operator $X: V_{1} \rightarrow V_{0}$ is an isomorphism decreasing the grading. In the general case we have

$$
X V_{k} \subset V_{0} \oplus V_{1} \oplus \cdots \oplus V_{k-1}, \quad k \geqslant 1 .
$$

We recall that according to formula $(5.9)$, the mapping $\left(D+q u_{1}\right)$ maps an element $F \in \operatorname{Ker} X^{m}$ into an element of a higher filtration from $\operatorname{Ker} X^{m+1}$.

The maximum eigenvalue $\lambda_{n}=\frac{n(n+1)}{2}$ of the operator $\left.X D\right|_{A_{n}}$ has multiplicity one, and the associated eigenvector is collinear to a homogeneous polynomial $P_{n}$ in $A_{n}$, which is given by an elegant formula similar to 5.2 for the Bell polynomials:

$$
P_{n}=\left(D+u_{1}\right)\left(D+2 u_{1}\right) \ldots\left(D+n u_{1}\right)(1) \text {. }
$$

In the examples, we have already seen the first polynomials from the sequence

$$
P_{1}=u_{1}, \quad P_{2}=u_{1}^{2}+u_{2}, \quad P_{3}=2 u_{1}^{2}+5 u_{1} u_{2}+u_{3}, \quad P_{4}=6 u_{1}^{4}+26 u_{1}^{2} u_{2}+9 u_{1} u_{3}+8 u_{2}^{2}+u_{4} .
$$

By induction, it is easy to find the values of the first and of the leading coefficients in the polynomial $P_{n}$

$$
P_{n}=(n-1) ! u_{1}^{n}+\cdots+u_{n}
$$

The existence of an eigenvector $P_{n}$ of multiplicity one with strictly positive coordinates for the operator $X D$ is an explicit manifestation of the classical Perron-Frobenius theorem on an operator with a matrix of a special form consisting of nonnegative elements.

We recall that, as in the Perron-Frobenius theorem, the eigenvalue $\lambda_{n}$ corresponding to the vector $P_{n}$ is positive and maximal among all eigenvalues of the operator $\left.X D\right|_{A_{n}}$. It is reasonable to assume that the family of polynomials $P_{n}$ not only has a nice recursive definition (5.14), but also has useful applications that have yet to be found.

\section{Characteristic Algebras FOR Systems OF RANK 2}

Similarly to the case of scalar hyperbolic equations, the concept of characteristic algebra can be extended to the case of an arbitrary exponential type system: following paper [1], a characteristic algebra of a system of form (1.1) is the Lie algebra generated by operators

$$
\frac{\partial}{\partial w^{1}}, \quad \frac{\partial}{\partial w^{2}}, \ldots, \frac{\partial}{\partial w^{r}}, \quad D_{y}
$$

where $D_{y}$ is the operator of total differentiation with respect to $y$ by virtue of the system. For exponential systems corresponding to non-degenerate Cartan matrices, the characteristic algebra is finite-dimensional [1].

We consider exponential systems corresponding to the Cartan matrices of affine Lie algebras of low rank $r$. It is easy to confirm that in the simplest case of rank 1 , the exponential systems corresponding to the Cartan matrices

$$
\left(\begin{array}{cc}
2 & -2 \\
-2 & 2
\end{array}\right) \text { and }\left(\begin{array}{cc}
2 & -4 \\
-1 & 2
\end{array}\right)
$$

are reduced respectively to the equations

$$
u_{x y}=e^{u}+e^{-u} \quad \text { and } \quad u_{x y}=e^{u}+e^{-2 u} \text {. }
$$


Both of these equations are well studied, the first of them is called sin-Gordon equation 1 , and the second is Tzizeica equation. The characteristic algebra of sine-Gordon equation was described explicitly in [21], and the characteristic algebra of Tzizeica equation was described in [25]. In papers [33], [34], another system of generators was constructed for these infinitedimensional Lie algebras and it was shown that they are isomorphic to the nonnegative parts of the loop algebra $\mathcal{L}(\mathfrak{s l}(2, \mathbb{C}))^{\geqslant 0}$ and twisted loop algebra $\mathcal{L}(\mathfrak{s l}(3, \mathbb{C}), \mu)^{\geqslant 0}$ respectively. Let us present these systems of generators in an explicit form.

For the equations of the form $u_{x y}=f(u)$, the characteristic algebra is generated by the operators $X_{0}=\frac{\partial}{\partial u}$ and by the operator $D_{y}$ of total differentiation by virtue of the equation. It is easy to check that if $f(u)=e^{u}+e^{\alpha u}$ as $\alpha \neq 0,1$, the $D_{y}=X_{1}+X_{2}$, where

$$
X_{1}=e^{u} \sum_{n=1}^{\infty} B_{n-1}(\mathbf{u}) \frac{\partial}{\partial u_{n}}, \quad X_{2}=e^{\alpha u} \sum_{n=1}^{\infty} B_{n-1}(\alpha \mathbf{u}) \frac{\partial}{\partial u_{n}},
$$

and $B_{n}(\mathbf{u})=B_{n}\left(u_{1}, \ldots, u_{n}\right)=e^{-u} D_{x}\left(e^{u}\right)$ is a complete Bell polynomial.

Theorem 6.1. [33], [34] The characteristic algebra $\chi_{s G}=\left\langle X_{0}, X_{1}, \ldots, X_{n}, \ldots\right\rangle$ of the sineGordon equation is generated by $X_{0}, X_{1}, X_{2}$ :

$$
X_{3 k+1}=-\left[X_{1}, X_{3 k}\right], \quad X_{3 k+2}=\left[X_{2}, X_{3 k}\right], \quad X_{3 k+3}=\left[X_{1}, X_{3 k+2}\right], \quad k=0,1,2, \ldots
$$

The characteristic algebra $\chi_{s G}$ is naturally graded: it can be represented as $\oplus_{i=0}^{+\infty} \mathfrak{g}_{i}$, where $\left[\mathfrak{g}_{1}, \mathfrak{g}_{i}\right]=\mathfrak{g}_{i+1}$. Indeed, using relations 6.1) and letting

$$
\mathfrak{g}_{0}=\left\langle X_{0}\right\rangle, \quad \mathfrak{g}_{1}=\left\langle X_{1}, X_{2}\right\rangle, \ldots, \mathfrak{g}_{2 k}=\left\langle X_{3 k}\right\rangle, \quad \mathfrak{g}_{2 k+1}=\left\langle X_{3 k+1}, X_{3 k+2}\right\rangle, \ldots,
$$

we obtain that the natural grading of the basis element $X_{n}$ is the number of commutators in its minimal representation by generators. Representing this as a diagram (see Figure 1), it is

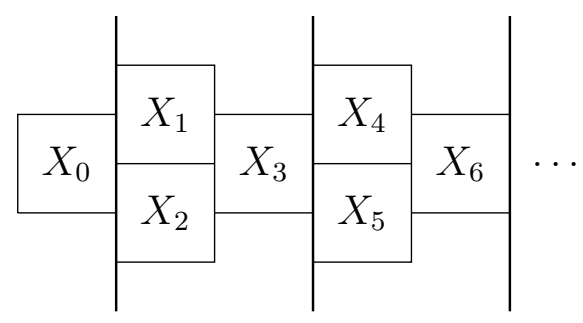

FIGURE 1. Characteristic algebra of sine-Gordon equation

easy to see that the average growth rate of this characteristic algebra, that is, the increase in dimension under adding one graded component, is equal to $\frac{3}{2}$.

Theorem 6.2. [33], [34] Characteristic algebra $\chi_{T z}=\left\langle X_{0}, X_{1}, \ldots, X_{n}, \ldots\right\rangle$ of the Tzizeica equation is generated by $X_{0}, X_{1}, X_{2}$ :

$X_{8 k+1}=-\left[X_{1}, X_{8 k}\right], \quad X_{8 k+2}=\frac{1}{2}\left[X_{2}, X_{8 k}\right], \quad X_{8 k+3}=\left[X_{1}, X_{8 k+2}\right], \quad X_{8 k+4}=\left[X_{1}, X_{8 k+3}\right]$,

$X_{8 k+5}=-\frac{1}{3}\left[X_{1}, X_{8 k+4}\right], \quad X_{8 k+6}=-\frac{1}{2}\left[X_{1}, X_{8 k+5}\right], \quad X_{8 k+7}=\left[X_{2}, X_{8 k+5}\right], \quad X_{8 k+8}=\left[X_{1}, X_{8 k+7}\right]$,

where $k=0,1,2, \ldots$

Representing the structure of this algebra in the form of a diagram, cf. Figure 2), it is easy to see that the rate of its growth is $\frac{4}{3}$.

\footnotetext{
${ }^{1}$ More precisely, it is more natural to call the equation $u_{x y}=\sin u$ the sine-Gordon equation, which is related with the equation we consider by a complex change. But since it is more convenient for us to work with an equation containing exponentials, we allow ourselves to admit such freedom in using the terminology.
} 


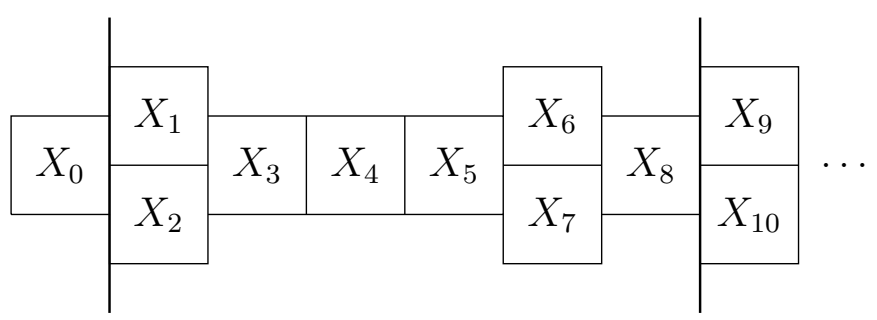

FIGURE 2. Characteristic algebra of Tzizeica equation

We proceed to studying the characteristic algebras for exponential systems corresponding to affine Cartan matrices of rank 2 (such matrices have size $3 \times 3$ ). The Cartan matrix

$$
\left(\begin{array}{ccc}
2 & -1 & -1 \\
-1 & 2 & -1 \\
-1 & -1 & 2
\end{array}\right)
$$

of the affine Lie algebra $A_{2}^{(1)}$ corresponds to the exponential system

$$
\left\{\begin{array}{l}
w_{1, x y}=\exp \left(2 w_{1}-w_{2}-w_{3}\right), \\
w_{2, x y}=\exp \left(-w_{1}+2 w_{2}-w_{3}\right), \\
w_{3, x y}=\exp \left(-w_{1}-w_{2}+2 w_{3}\right) .
\end{array}\right.
$$

The change of variable $u=2 w_{1}-w_{2}-w_{3}, v=-w_{1}+2 w_{2}-w_{3}$ reduces this system to

$$
\left\{\begin{array}{l}
u_{x y}=2 e^{u}-e^{v}-e^{-u-v} \\
v_{x y}=-e^{u}+2 e^{v}-e^{-u-v} .
\end{array}\right.
$$

The operator $D_{y}$ of total differentiation by virtue of system 6.2 reads as

$$
D_{y}=X_{1}+X_{2}+X_{3},
$$

where

$$
\begin{aligned}
& X_{1}=e^{u} \sum_{n=1}^{\infty} B_{n-1}(\mathbf{u})\left(2 \frac{\partial}{\partial u_{n}}-\frac{\partial}{\partial v_{n}}\right), \\
& X_{2}=e^{v} \sum_{n=1}^{\infty} B_{n-1}(\mathbf{v})\left(-\frac{\partial}{\partial u_{n}}+2 \frac{\partial}{\partial v_{n}}\right), \\
& X_{3}=-e^{-u-v} \sum_{n=1}^{\infty} B_{n-1}(-\mathbf{u}-\mathbf{v})\left(\frac{\partial}{\partial u_{n}}+\frac{\partial}{\partial v_{n}}\right) .
\end{aligned}
$$

Theorem 6.3. The characteristic algebra $\chi_{A_{2}^{(1)}}=\left\langle X_{0}, X_{1}, \ldots, X_{n}, \ldots\right\rangle$ of system 6.2 is generated by $Y_{0}=\frac{\partial}{\partial u}, Y_{0}^{\prime}=\frac{\partial}{\partial v}, X_{1}, X_{2}, X_{3}$ :

$$
\begin{aligned}
& X_{8 k+1}=\left[X_{1}, X_{8 k-1}\right], \quad X_{8 k+2}=-\left[X_{2}, X_{8 k}\right], \quad X_{8 k+3}=\left[X_{3}, X_{8 k-1}\right], \quad X_{8 k+4}=\left[X_{1}, X_{8 k+2}\right], \\
& X_{8 k+5}=\left[X_{1}, X_{8 k+3}\right], \quad X_{8 k+6}=\left[X_{2}, X_{8 k+3}\right], \quad X_{8 k+7}=\left[X_{2}, X_{8 k+5}\right], \quad X_{8 k+8}=\left[X_{1}, X_{8 k+6}\right],
\end{aligned}
$$
where $X_{-1}=-2 Y_{0}+Y_{0}^{\prime}, X_{0}=-Y_{0}+2 Y_{0}^{\prime}$ and $k=0,1,2, \ldots$

It is easy to see that the average growth rate of the characteristic algebra of system $(6.2)$ is equal to $\frac{8}{3}$.

The proof is based on the following two simple lemmata; the first of them goes back to A.B. Shabat. Let

$$
D=u_{1} \frac{\partial}{\partial u}+v_{1} \frac{\partial}{\partial v}+u_{2} \frac{\partial}{\partial u_{2}}+v_{2} \frac{\partial}{\partial v_{2}}+\ldots
$$




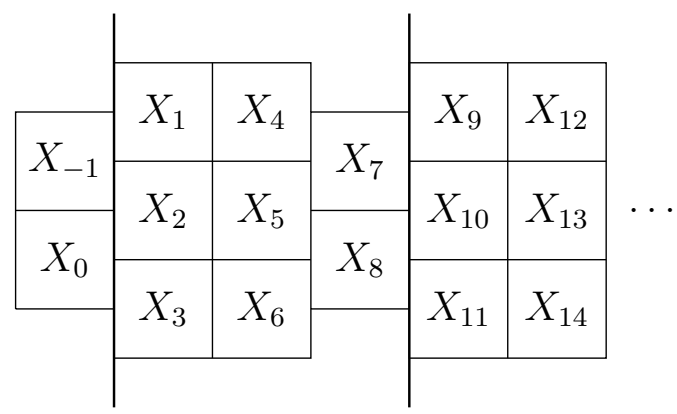

FiguRE 3. Characteristic algebra of system $A_{2}^{(1)}$

to simplify the formulae, we keep the notation $D$ for the operators of form more general than in Section 3 .

Lemma 6.1. If the differential operator

$$
X=\sum_{n=1}^{+\infty}\left(P_{n}\left(u, v, u_{1}, v_{1}, u_{2}, v_{2} \ldots\right) \frac{\partial}{\partial u_{n}}+Q_{n}\left(u, v, u_{1}, v_{1}, u_{2}, v_{2} \ldots\right) \frac{\partial}{\partial v_{n}}\right)
$$

where $P_{n}$ and $Q_{n}$ are polynomials, satisfies the relation $[D, X]=0$, then $X=0$.

The next lemma generalizes Lemma 5.2 in Section 5 .

Lemma 6.2. Let $f=f\left(u_{1}, v_{1}, u_{2}, v_{2} \ldots\right)$ be a function depending on finitely many variables and $c$ be an arbitrary constant. Then for arbitrary constants $\alpha$ and $\beta$ the equation

$$
\left(D+\alpha u_{1}+\beta v_{1}\right) f=c
$$

has no solutions as $c \neq 0$ and it possesses only the trivial solution $f \equiv 0$ as $c=0$.

Proof. Since $\left[Y_{0}, D_{y}\right]=\left[Y_{0}, X_{1}+X_{2}+X_{3}\right]=X_{1}-X_{3}$, the operator $X_{1}-X_{3}$ belongs to the characteristic algebra. Then $\left[Y_{0}^{\prime}, X_{1}-X_{3}\right]=X_{3}$, which implies that $X_{3}$, and hence, $X_{1}$, also belongs to the characteristic algebra. This is why

$$
X_{2}=D_{y}-X_{1}-X_{3} \in \chi_{A_{2}^{(1)}} .
$$

Thus, the characteristic algebra is generated by the elements $Y_{0}, Y_{0}^{\prime}, X_{1}, X_{2}$ and $X_{3}$.

It follows from the Jacobi identity that all higher order commutators are expressed as linear combinations of commutators of the form $[A,[B,[C,[\ldots]]]]$, where $A, B, C, \ldots$ are the generators. Thus, it is sufficient to consider only commutators of such type. The further proof is similar to the proof of Theorems 6.1 and 6.2 in paper [34] and is carried out by induction in $k$, where $k$ is the number of periodically repeating groups of 8 elements, see Figure 3 . First, considering the commutation relations of the generators and their commutators with elements $Y_{0}, Y_{0}^{\prime}$ and $D$ and using Lemma 6.1, we can show that among all of the commutators of the natural grading 2,3 , the only nontrivial elements are $X_{4}, X_{5}, \ldots, X_{8}$. The independence of all elements $X_{1}, X_{2}, \ldots, X_{8}$ can be proved by contradiction using Lemmata 6.1, 6.2. This completes the induction base.

The induction step is based on two key ideas. First, the commutation relations within one group $X_{8 k+1}, X_{8 k+2}, \ldots, X_{8 k+8}$ imply similar commutation relations in the next group. Hence, using Lemma 6.1, one can deduce the triviality of those commutators that are not listed in the table. Second, the independence of the others is deduced from the independence proved at the previous step by successively adding new elements (using Lemmata 6.1, 6.2), which completes the induction step. 
We proceed to studying characteristic algebras of systems corresponding to other affine Lie algebras. We consider the Cartan matrix

$$
\left(\begin{array}{ccc}
2 & -1 & 0 \\
-2 & 2 & -2 \\
0 & -1 & 2
\end{array}\right)
$$

of affine Lie algebra $B_{2}^{(2)}$; it corresponds to the exponential system

$$
\left\{\begin{array}{l}
w_{1, x y}=\exp \left(2 w_{1}-w_{2}\right) \\
w_{2, x y}=\exp \left(-2 w_{1}+2 w_{2}-2 w_{3}\right), \\
w_{3, x y}=\exp \left(-w_{2}+2 w_{3}\right)
\end{array}\right.
$$

Under the change of variables $u=2 w_{1}-w_{2}, v=-2 w_{1}+2 w_{2}-2 w_{3}$ this system is reduced to

$$
\left\{\begin{array}{l}
u_{x y}=2 e^{u}-e^{v}, \\
v_{x y}=-2 e^{u}+2 e^{v}-2 e^{-u-v} .
\end{array}\right.
$$

The operator $D_{y}$ of total differentiation by virtue of system 6.3 is as follows

$$
D_{y}=X_{1}+X_{2}+X_{3}
$$

where

$$
\begin{aligned}
& X_{1}=e^{u} \sum_{n=1}^{\infty} B_{n-1}(\mathbf{u})\left(2 \frac{\partial}{\partial u_{n}}-2 \frac{\partial}{\partial v_{n}}\right) \\
& X_{2}=e^{v} \sum_{n=1}^{\infty} B_{n-1}(\mathbf{v})\left(-\frac{\partial}{\partial u_{n}}+2 \frac{\partial}{\partial v_{n}}\right), \\
& X_{3}=-2 e^{-u-v} \sum_{n=1}^{\infty} B_{n-1}(-\mathbf{u}-\mathbf{v}) \frac{\partial}{\partial v_{n}} .
\end{aligned}
$$

Theorem 6.4. The characteristic algebra $\chi_{B_{2}^{(2)}}=\left\langle X_{0}, X_{1}, \ldots, X_{n}, \ldots\right\rangle$ of system 6.3 is generated by $Y_{0}=\frac{\partial}{\partial u}, Y_{0}^{\prime}=\frac{\partial}{\partial v}, X_{1}, X_{2}, X_{3}$ :

$$
\begin{aligned}
& X_{15 k+1}=-\left[X_{1}, X_{15 k-1}\right], \quad X_{15 k+2}=\left[X_{2}, X_{15 k}\right], \quad X_{15 k+3}=-\left[X_{3}, X_{15 k}\right], \\
& X_{15 k+4}=\left[X_{1}, X_{15 k+2}\right], \quad X_{15 k+5}=\left[X_{2}, X_{15 k+3}\right], \quad X_{15 k+6}=\left[X_{1}, X_{15 k+4}\right] \text {, } \\
& X_{15 k+7}=\left[X_{1}, X_{15 k+5}\right], \quad X_{15 k+8}=\left[X_{3}, X_{15 k+5}\right], \quad X_{15 k+9}=\left[X_{1}, X_{15 k+7}\right] \text {, } \\
& X_{15 k+10}=\left[X_{1}, X_{15 k+8}\right], \quad X_{15 k+11}=\left[X_{1}, X_{15 k+10}\right], \quad X_{15 k+12}=\left[X_{2}, X_{15 k+9}\right] \text {, } \\
& X_{15 k+13}=\left[X_{2}, X_{15 k+10}\right], \quad X_{15 k+14}=\left[X_{1}, X_{15 k+13}\right], \quad X_{15 k+15}=\left[X_{3}, X_{15 k+12}\right],
\end{aligned}
$$

where $X_{-1}=Y_{0}-Y_{0}^{\prime}, X_{0}=-Y_{0}^{\prime}$ and $k=0,1,2, \ldots$ $\frac{5}{2}$.

It is easy to see that the average growth rate of the characteristic algebra of system $(6.3)$ is

We consider the Cartan matrix

$$
\left(\begin{array}{ccc}
2 & -2 & 0 \\
-1 & 2 & -2 \\
0 & -1 & 2
\end{array}\right)
$$

of affine Lie algebra $\tilde{B}_{2}^{(2)}$; it corresponds to the exponential system

$$
\left\{\begin{array}{l}
w_{1, x y}=\exp \left(2 w_{1}-2 w_{2}\right) \\
w_{2, x y}=\exp \left(-w_{1}+2 w_{2}-2 w_{3}\right) \\
w_{3, x y}=\exp \left(-w_{2}+2 w_{3}\right)
\end{array}\right.
$$




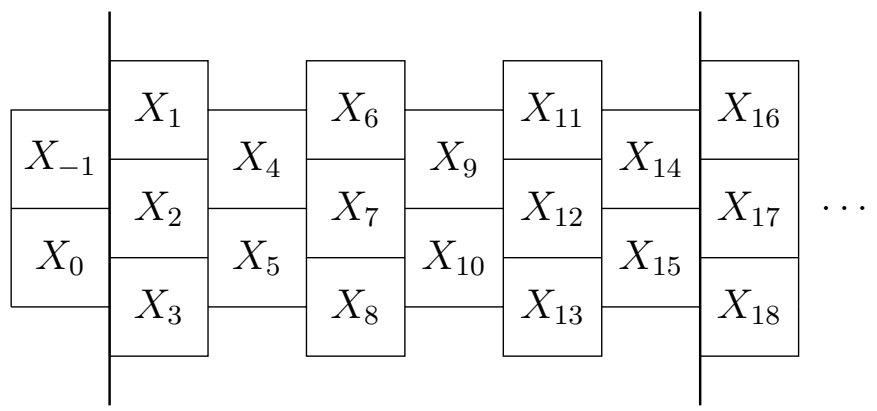

FIGURE 4. Characteristic algebra of system $B_{2}^{(2)}$

Under the change of the variables $u=2 w_{1}-2 w_{2}, v=-w_{1}+2 w_{2}-2 w_{3}$ this system is reduced to

$$
\left\{\begin{array}{l}
u_{x y}=2 e^{u}-2 e^{v} \\
v_{x y}=-e^{u}+2 e^{v}-2 e^{-\frac{u}{2}-v} .
\end{array}\right.
$$

The operator $D_{y}$ of total differentiation by virtue of system 6.4 reads as

$$
D_{y}=X_{1}+X_{2}+X_{3},
$$

where

$$
\begin{aligned}
& X_{1}=e^{u} \sum_{n=1}^{\infty} B_{n-1}(\mathbf{u})\left(2 \frac{\partial}{\partial u_{n}}-\frac{\partial}{\partial v_{n}}\right), \\
& X_{2}=e^{v} \sum_{n=1}^{\infty} B_{n-1}(\mathbf{v})\left(-2 \frac{\partial}{\partial u_{n}}+2 \frac{\partial}{\partial v_{n}}\right), \\
& X_{3}=-2 e^{-\frac{u}{2}-v} \sum_{n=1}^{\infty} B_{n-1}\left(-\frac{\mathbf{u}}{2}-\mathbf{v}\right) \frac{\partial}{\partial v_{n}} .
\end{aligned}
$$

Theorem 6.5. The characteristic algebra $\chi_{\tilde{B}_{2}^{(2)}}=\left\langle X_{0}, X_{1}, \ldots, X_{n}, \ldots\right\rangle$ of system (6.4) is generated by $Y_{0}=\frac{\partial}{\partial u}, Y_{0}^{\prime}=\frac{\partial}{\partial v}, X_{1}, X_{2}, X_{3}$ :

$$
\begin{aligned}
& X_{24 k+1}=-\frac{1}{2}\left[X_{1}, X_{24 k-1}\right], \quad X_{24 k+2}=2\left[X_{2}, X_{24 k}\right], \quad X_{24 k+3}=-2\left[X_{3}, X_{24 k}\right], \\
& X_{24 k+4}=\left[X_{1}, X_{24 k+2}\right], \quad X_{24 k+5}=\left[X_{2}, X_{24 k+3}\right], \quad X_{24 k+6}=\left[X_{1}, X_{24 k+5}\right], \\
& X_{24 k+7}=\left[X_{2}, X_{24 k+4}\right], \quad X_{24 k+8}=\left[X_{3}, X_{24 k+5}\right], \quad X_{24 k+9}=\left[X_{1}, X_{24 k+8}\right], \\
& X_{24 k+10}=\left[X_{2}, X_{24 k+6}\right], \quad X_{24 k+11}=\left[X_{2}, X_{24 k+9}\right], \quad X_{24 k+12}=\left[X_{3}, X_{24 k+10}\right] \text {, } \\
& X_{24 k+13}=\left[X_{2}, X_{24 k+12}\right], \quad X_{24 k+14}=\left[X_{3}, X_{24 k+12}\right], \quad X_{24 k+15}=\left[X_{1}, X_{24 k+13}\right], \\
& X_{24 k+16}=\left[X_{2}, X_{24 k+14}\right], \quad X_{24 k+17}=\left[X_{3}, X_{24 k+14}\right], \quad X_{24 k+18}=\left[X_{1}, X_{24 k+16}\right], \\
& X_{24 k+19}=\left[X_{3}, X_{24 k+16}\right], \quad X_{24 k+20}=\left[X_{1}, X_{24 k+19}\right], \quad X_{24 k+21}=\left[X_{2}, X_{24 k+18}\right] \text {, } \\
& X_{24 k+22}=\left[X_{2}, X_{24 k+19}\right], \quad X_{24 k+23}=\left[X_{1}, X_{24 k+22}\right], \quad X_{24 k+24}=\left[X_{3}, X_{24 k+21}\right],
\end{aligned}
$$

where $X_{-1}=2 Y_{0}-Y_{0}^{\prime}, X_{0}=-\frac{1}{2} Y_{0}^{\prime}$ and $k=0,1,2, \ldots$

It is easy to see that the average growth rate of the characteristic algebra of system $(6.4)$ is equal to $\frac{12}{5}$.

We consider the Cartan matrix

$$
\left(\begin{array}{ccc}
2 & -2 & 0 \\
-1 & 2 & -1 \\
0 & -2 & 2
\end{array}\right)
$$




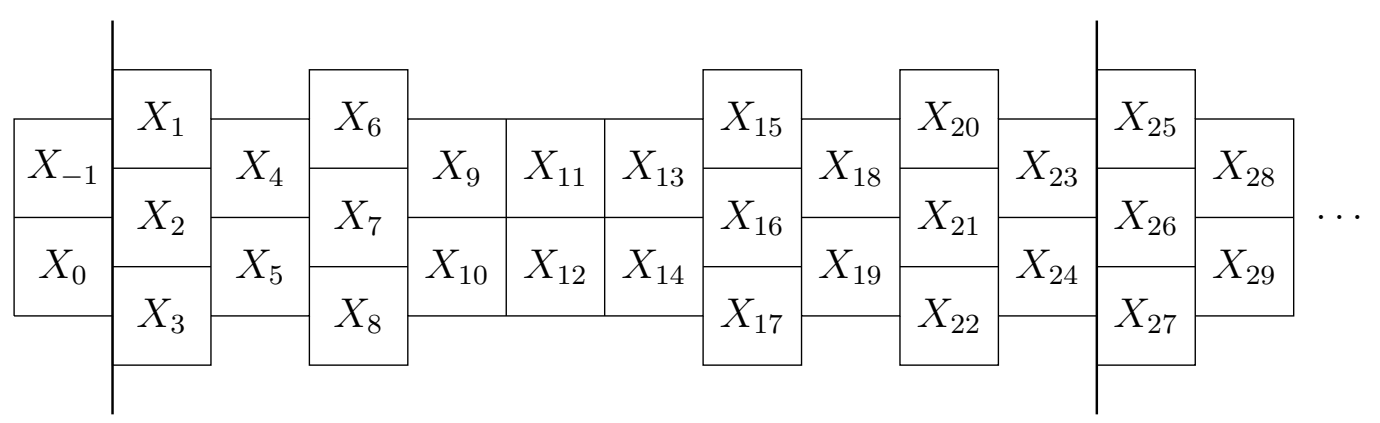

FIGURE 5. Characteristic algebra of system $\tilde{B}_{2}^{(2)}$

of affine Lie algebra $C_{2}^{(1)}$; it corresponds to the exponential system

$$
\left\{\begin{array}{l}
w_{1, x y}=\exp \left(2 w_{1}-2 w_{2}\right) \\
w_{2, x y}=\exp \left(-w_{1}+2 w_{2}-w_{3}\right) \\
w_{3, x y}=\exp \left(-2 w_{2}+2 w_{3}\right)
\end{array}\right.
$$

By the change of variables $u=2 w_{1}-2 w_{2}, v=-w_{1}+2 w_{2}-w_{3}$ this system is reduced to

$$
\left\{\begin{array}{l}
u_{x y}=2 e^{u}-2 e^{v}, \\
v_{x y}=-e^{u}+2 e^{v}-e^{-u-2 v} .
\end{array}\right.
$$

The operator $D_{y}$ of total differentiation by virtue of system reads as

$$
D_{y}=X_{1}+X_{2}+X_{3},
$$

where

$$
\begin{aligned}
& X_{1}=e^{u} \sum_{n=1}^{\infty} B_{n-1}(\mathbf{u})\left(2 \frac{\partial}{\partial u_{n}}-\frac{\partial}{\partial v_{n}}\right), \\
& X_{2}=e^{v} \sum_{n=1}^{\infty} B_{n-1}(\mathbf{v})\left(-2 \frac{\partial}{\partial u_{n}}+2 \frac{\partial}{\partial v_{n}}\right), \\
& X_{3}=-e^{-u-2 v} \sum_{n=1}^{\infty} B_{n-1}(-\mathbf{u}-2 \mathbf{v}) \frac{\partial}{\partial v_{n}} .
\end{aligned}
$$

Theorem 6.6. Characteristic algebra $\chi_{C_{2}^{(1)}}=\left\langle X_{0}, X_{1}, \ldots, X_{n}, \ldots\right\rangle$ of system 6.5) is generated by $Y_{0}=\frac{\partial}{\partial u}, Y_{0}^{\prime}=\frac{\partial}{\partial v}, X_{1}, X_{2}, X_{3}$ :

$$
\begin{aligned}
& X_{10 k+1}=\left[X_{1}, X_{10 k}\right], \quad X_{10 k+2}=-\left[X_{2}, X_{10 k-1}\right], \quad X_{10 k+3}=\left[X_{3}, X_{10 k}\right], \\
& X_{10 k+4}=\left[X_{1}, X_{10 k+2}\right], \quad X_{10 k+5}=\left[X_{2}, X_{10 k+3}\right], \quad X_{10 k+6}=\left[X_{1}, X_{10 k+5}\right], \\
& X_{10 k+7}=\left[X_{2}, X_{10 k+4}\right], \quad X_{10 k+8}=\left[X_{2}, X_{10 k+5}\right], \quad X_{10 k+9}=\left[X_{1}, X_{10 k+8}\right], \\
& X_{10 k+10}=\left[X_{2}, X_{10 k+6}\right] \text {, }
\end{aligned}
$$

where $X_{-1}=Y_{0}^{\prime}-2 Y_{0}, X_{0}=Y_{0}^{\prime}-Y_{0}$ and $k=0,1,2, \ldots$

It is easy to see that the average growth rate of the characteristic algebra of system $(6.5)$ is equal to $\frac{5}{2}$.

The proof of Theorems 6.4 6.6 is similar to the proof of Theorem 6.3 . 


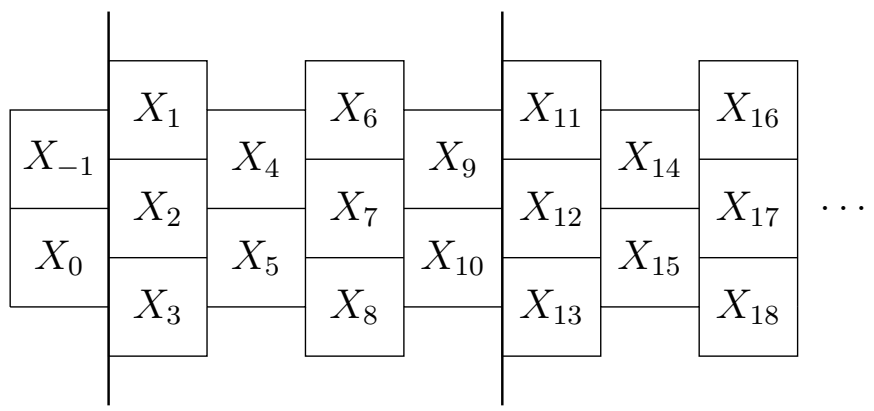

FIGURE 6. Characteristic algebra of the system $C_{2}^{(1)}$

\section{Characteristic Algebras AND Symmetries}

It is well known (see, for example, [31]) that both the sine-Gordon and Tzitzeica equations have an infinite hierarchy of higher symmetries. Let us formulate the corresponding results.

Theorem 7.1. [31] The equation $u_{x y}=e^{u}+e^{-u}$ has an infinite hierarchy of homogeneous graded polynomial symmetries $u_{t}=F_{k}\left(u_{1}, u_{2}, u_{3}, \ldots\right)$, where $k=1,3,5,7, \ldots$ and

$$
F_{2 k+1}=L^{k}\left(u_{1}\right), \quad L=\left(D+u_{1}\right)\left(D-u_{1}+D^{-1} u_{2}\right) .
$$

All polynomial symmetries of form $u_{t}=F\left(u_{1}, u_{2}, u_{3}, \ldots\right)$ are linear combinations with constant coefficients of symmetries $F_{1}, F_{3}, F_{5}, \ldots$

Theorem 7.2. [31] The equation $u_{x y}=e^{u}+e^{-2 u}$ has an infinite hierarchy of homogeneous graded polynomial symmetries

$$
u_{t}=F_{6 k+1}\left(u_{1}, u_{2}, u_{3}, \ldots\right) \quad \text { and } \quad u_{t}=F_{6 k+5}\left(u_{1}, u_{2}, u_{3}, \ldots\right),
$$

where $k=0,1,2, \ldots$ and

$$
\begin{aligned}
F_{6 k+1} & =L^{k}\left(u_{1}\right), \quad F_{6 k+5}=L^{k}\left(u_{5}+5\left(u_{2}-u_{1}^{2}\right) u_{3}-5 u_{1} u_{2}^{2}+u_{1}^{5}\right), \\
L & =\left(D-u_{1}-2 u_{1} D^{-1} u_{1}\right)\left(D-u_{1}\right) D\left(D+u_{1}\right)\left(D^{2}+u_{1} D-2 u_{1}^{2}+2 u_{1} D^{-1} u_{2}\right) .
\end{aligned}
$$

All polynomial symmetries of form $u_{t}=F\left(u_{1}, u_{2}, u_{3}, \ldots\right)$ are linear combinations with constant coefficients of symmetries $F_{6 k+1}, F_{6 k+5}$.

There are two important points to be noted. First, in both cases, the structure of the symmetries turns out to be related with the structure of naturally graded components of the corresponding characteristic algebras: for the sine-Gordon equation, there is a periodicity with period 2, and for the Tzizeica equation the period is 6 ; the symmetries are numbered according their graduation. Second, despite the fact that the recursion operators $L$ in each of the cases contain pseudodifferential components $D^{-1}$, the symmetries are arranged in such a way that when the operator $L$ is applied to them, the nonlocality disappears.

A similar connection between the hierarchy of symmetries and the structure of naturally graded components of the corresponding characteristic algebra is also observed for exponential systems of rank 2 .

Theorem 7.3. Exponential system 6.2) corresponding to the Cartan matrix of the affine Lie algebra $A_{2}^{(1)}$ has an infinite hierarchy of graded homogeneous polynomial symmetries

$$
\begin{array}{ll}
u_{t}=F_{3 k+1}\left(u_{1}, v_{1}, u_{2}, v_{2}, \ldots\right), & v_{t}=G_{3 k+1}\left(u_{1}, v_{1}, u_{2}, v_{2}, \ldots\right), \\
u_{t}=F_{3 k+2}\left(u_{1}, v_{1}, u_{2}, v_{2}, \ldots\right), & v_{t}=G_{3 k+2}\left(u_{1}, v_{1}, u_{2}, v_{2}, \ldots\right),
\end{array}
$$


where

$$
\left(\begin{array}{c}
F_{3 k+1} \\
G_{3 k+1}
\end{array}\right)=L^{k}\left(\begin{array}{l}
u_{1} \\
v_{1}
\end{array}\right), \quad\left(\begin{array}{c}
F_{3 k+2} \\
G_{3 k+2}
\end{array}\right)=L^{k}\left(\begin{array}{c}
u_{2}+2 v_{2}+u_{1}^{2}+2 u_{1} v_{1} \\
-2 u_{2}-v_{2}-v_{1}^{2}-2 u_{1} v_{1}
\end{array}\right)
$$

and the operator $L$ is defined by the following formula

$$
\begin{aligned}
L= & \left(\begin{array}{cc}
1 & 2 \\
-2 & -1
\end{array}\right) D^{3}+2\left(\begin{array}{cc}
u_{1}+v_{1} & u_{1} \\
-v_{1} & -u_{1}-v_{1}
\end{array}\right) D^{2} \\
& +\left(\left(\begin{array}{cc}
u_{2}+v_{2} & u_{2} \\
-v_{2} & -u_{2}-v_{2}
\end{array}\right)-\frac{A}{3}\left(\begin{array}{cc}
1 & 2 \\
-2 & -1
\end{array}\right)\right) D-\left(\begin{array}{cc}
u_{1} & 0 \\
0 & v_{1}
\end{array}\right) D^{-1}\left(\begin{array}{cc}
B & C \\
B & C
\end{array}\right) D \\
& +\frac{1}{3}\left(\begin{array}{cc}
u_{2}+2 v_{2}+2 u_{1} v_{1}+u_{1}^{2} & 0 \\
0 & v_{2}+2 u_{2}+2 u_{1} v_{1}+v_{1}^{2}
\end{array}\right) D^{-1}\left(\begin{array}{cc}
-2 u_{1}-v_{1} & -u_{1}-2 v_{1} \\
2 u_{1}+v_{1} & u_{1}+2 v_{1}
\end{array}\right) D \\
A & =u_{1}^{2}+u_{1} v_{1}+v_{1}^{2}, \quad B=\frac{2}{3} u_{1}^{2}-\frac{1}{3} v_{1}^{2}+\frac{2}{3} u_{1} v_{1}+v_{2}, \quad C=\frac{1}{3} u_{1}^{2}-\frac{2}{3} v_{1}^{2}-\frac{2}{3} u_{1} v_{1}-u_{2} .
\end{aligned}
$$

All polynomial symmetries of form $u_{t}=F\left(u_{1}, v_{1}, u_{2}, v_{2}, \ldots\right), v_{t}=G\left(u_{1}, v_{1}, u_{2}, v_{2}, \ldots\right)$ are linear combinations with constant coefficients of the symmetriues $\left(F_{3 k+1}, G_{3 k+1}\right),\left(F_{3 k+2}, G_{3 k+2}\right)$.

Proof. For the sake of convenience we introduce the notation

$$
X=e^{-u} X_{1}, \quad Y=e^{-v} X_{2}, \quad Z=e^{u+v} X_{3} .
$$

It is easy to check that the relations

$$
\left(D+u_{1}\right) X=X D, \quad\left(D+v_{1}\right) Y=Y D, \quad\left(D-u_{1}-v_{1}\right) Z=Z D
$$

hold and that the equations $u_{t}=F, v_{t}=G$ define a symmetry of system $(6.2)$ if and only if the following conditions are satisfied:

$$
X D\left(\begin{array}{l}
F \\
G
\end{array}\right)=\left(\begin{array}{c}
2 F \\
-F
\end{array}\right), \quad Y D\left(\begin{array}{l}
F \\
G
\end{array}\right)=\left(\begin{array}{c}
-G \\
2 G
\end{array}\right), \quad Z D\left(\begin{array}{l}
F \\
G
\end{array}\right)=\left(\begin{array}{l}
F+G \\
F+G
\end{array}\right)
$$

Let a pair of homogeneous graded polynomials $\tilde{F}$ and $\tilde{G}$ of the same degree define a symmetry of system 6.2. Then, using relations 7.2 , one can show that the functions

$$
F=X Z Y \tilde{F} \quad \text { and } \quad G=Y X Z \tilde{G}
$$

satisfy conditions 7.3 , i.e., they also define a symmetry of system 6.2 . Since applying of each of the operators $X, Y$ and $Z$ to a homogeneous polynomial decreases its grading by one, the grading of the symmetry $(F, G)$ is less than the grading of $\tilde{F}, \tilde{G}$ by 3 . It is straightforward to confirm that the only (up to a rescaling by a constant) symmetries of the first and second order of system 6.2 are respectively

$$
u_{t}=F_{1}, \quad v_{t}=G_{1} \quad \text { and } \quad u_{t}=F_{2}, \quad v_{t}=G_{2} .
$$

In the same way it is easy to show that system (6.2) has no third order symmetries, which implies that it also cannot have symmetries of order $3 k$, where $k \in \mathbb{N}$. Further, by rather cumbersome calculations using identities 7.2 , we can invert formulae 7.3):

$$
\left(\begin{array}{l}
\tilde{F} \\
\tilde{G}
\end{array}\right)=\frac{1}{9} \cdot L\left(\begin{array}{l}
F \\
G
\end{array}\right),
$$

where the operator $L$ is defined by formula $(7.1)$. Thus, each of the symmetries $\left(F_{1}, G_{1}\right)$ and $\left(F_{2}, G_{2}\right)$ uniquely (up to a rescaling by a constant) generates a sequence of symmetries in the gradings $3 k+1$ and $3 k+2$, respectively. 
The structure of symmetries for systems (6.3)-(6.5) looks similar. A computer experiment shows that system (6.3) has one symmetry in the weights $1,3,5,7$, and in the weights 2,4 , 6 there are no symmetries (we do not provide explicit expressions for symmetries since they are too bulky). It is not difficult to construct operators like (7.3) that map symmetries into symmetries and decrease the weight by 6 . Hence, in particular, it follows that system (6.3) has no symmetries in even weights.

System (6.5) has one symmetry in weights $1,3,5,7$ and has no symmetries in weights $2,4,6$. Since for this system there is an operator that maps symmetries into symmetries and increases the weight by 4 , it follows that it has no symmetries in even weights. For system (6.4), we succeed to find symmetries in the weights 1,3, 7 and show that they are absent in the weights $2,4,5,6$. For this system, there is an operator that maps symmetries into symmetries and decreases the weight by 10 . It is easy to see that these results completely repeat the structure of characteristic algebras for the corresponding systems, see Figures 3 ,6. The above observations allow us to formulate the following conjecture.

Conjecture 7.1. The characteristic algebra of an exponential system corresponding to the Cartan matrix of an arbitrary affine Lie algebra, has a linear growth and the average rate of this growth does not exceed the size $r+1$ of the corresponding Cartan matrix, where $r$ is the rank of this matrix. The naturally graded structure of such characteristic algebra of the system is periodic with some period $m \in \mathbb{N}$. Such system has an infinite hierarchy of symmetries homogeneous in grading, which consists finitely many sequences. All elements of such sequence are of the form $L^{k}(\mathbf{F})$, where $L$ is some linear operator, $k=1,2, \ldots$ and $\mathbf{F}=\left(F^{1}, \ldots, F^{r}\right)$ is an initial symmetry of order less than $m$. Any symmetry depending polynomially on the $x$-derivatives of dynamical variables is a linear combination with constant coefficients of the above described symmetries.

\section{ACKNOWLEDGMENTS}

The authors express their gratitude to A.V. Zhiber, O.V. Kaptsov, M.V. Pavlov, V.V. Sokolov, E.V. Ferapontov and I.T. Habibullin for useful discussion which stimulated a lot the present work.

\section{BIBLIOGRAPHY}

1. A.B. Shabat, R.I. Yamilov. Exponential systems of type I and Cartan matrices // Preprint. Bashkir Branch of Academy of Sciences of USSR. (1981). (in Russian).

2. A.N. Leznov, V.G. Smirnov, A.B. Shabat. The group of internal symmetries and the conditions of integrability of two-dimensional dynamical systems // Teor. Matem. Fiz. 51:1, 10-21 (1982). [Theor. Math. Phys. 51:1, 322-330 (1982).]

3. A.N. Leznov. On the complete integrability of a nonlinear system of partial differential equations in two-dimensional space // Teor. Matem. Fiz. 42:3, 343-349 (1980). [Theor. Math. Phys. 42:3, 225-229 (1980).]

4. V.E. Adler, I.T. Habibullin. Integrable boundary conditions for the Toda lattice // J. Phys. A: Math. Gen. 28:23, 6711-6129 (1995).

5. B. Gürel, I. Habibullin. Boundary conditions for two-dimensional integrable chains // Phys. Lett. A. 233:3-4, 68-72 (1997).

6. I.T. Habibullin. Truncations of Toda chains and the reduction problem // Teor. Matem. Fiz. 143:1, 22-48 (2005). [Theor. Math. Phys. 143:1, 515-528 (2005).]

7. R.S. Ward. Discrete Toda field equations // Phys. Lett. A. 199:1-2, 45-48 (1995).

8. A. Doliwa. Geometric discretisation of the Toda system // Phys. Lett. A. 234:3, 187-192 (1997).

9. I.T. Habibullin. C-series discrete chains // Teor. Matem. Fiz. 146:2, 208-221 (2006). [Theor. Math. Phys. 146:2, 170-182 (2006).] 
10. S.V. Smirnov. Semidiscrete Toda lattices // Teor. Matem. Fiz. 172:3, 387-404 (2012). [Theor. Math. Phys. 172:3, 1217-1231 (2012).]

11. I. Habibullin, K. Zheltukhin, M. Yangubaeva. Cartan matrices and integrable lattice Toda field equations // J. Phys. A: Math. Theor. 44:46, 465202 (2011).

12. R. Garifullin, I. Habibullin, M. Yangubaeva. Affine and finite Lie algebras and integrable Toda field equations on discrete time-space // SIGMA. 8:062 (2012).

13. S.V. Smirnov. Darboux integrability of discrete two-dimensional Toda lattices // Teor. Matem. Fiz. 182:5, 228-253 (2015). [Theor. Math. Phys. 182:2, 189-210 (2015).]

14. D.K. Demskoi, D.T. Tran. Darboux integrability of determinant and equations for principal minors // Nonlinearity. 29:7, 1973-1991 (2016).

15. I.T. Habibullin, A.R. Khakimova. Discrete exponential type systems on a quad graph, corresponding to the affine Lie algebras $A_{N-1}^{(1)} / /$ J. Phys. A: Math. Theor. 52:36, 365202 (2019).

16. I. Habibullin, A. Khakimova. Integrable boundary conditions for the Hirota-Miwa equation and Lie algebras // J. Nonl. Math. Phys. 27:3, 393-413 (2020).

17. I.T. Habibullin, M.N. Kuznetsova, A.U. Sakieva. Integrability conditions for two-dimensional Toda-like equations // J. Phys. A: Math. Theor. 53:39, 395203 (2020).

18. E. Goursat. Recherches sur quelques équations aux dérivées partielles du second ordre // Ann. Fac. Sci. Toulouse. 1, 31-78, 439-464 (1899).

19. A.V. Zhiber, N.Kh. Ibragimov, A.B. Shabat. Equations of Liouville type // Dokl. AN SSSR. 249:1, 26-29 (1979). [Sov. Math. Dokl. 20, 1183-1187 (1979).]

20. I.T. Habibullin. Characteristic algebras of fully discrete hyperbolic type equations // SIGMA. 1:023 (2005).

21. A.V. Zhiber, R.D. Murtazina. On the characteristic Lie algebras for equations $u_{x y}=f\left(u, u_{x}\right) / /$ Fundam. Prikl. Matem. 12:7, 65-78 (2006). [J. Math. Sci. 151:4, 3112-3122 (2008).]

22. I.T. Habibullin, A. Pekcan. Characteristic Lie algebra and classification of semidiscrete models // Teor. Matem. Fiz. 151:3, 413-423 (2007). [Theor. Math. Phys. 151:3, 781-790 (2007).]

23. A.V. Zhiber, O.S. Kostrigina. Characteristic algebras of nonlinear hyperbolic systems of equations // J. Sib. Fed. Univ. Math. Phys. 3:2, 173-184 (2010). (in Russian).

24. N.A. Zheltukhina, A.U. Sakieva, I.T. Habibullin. Characteristic Lie algebra and Darboux integrable discrete chains // Ufimskji Matem. Zhurn. 2:4, 39-51 (2010). (in Russian).

25. A.U. Sakieva. Characteristic Lie ring of the Zhiber-Shabat-Tzitzeica equation// Ufimskij Matem. Zhurn. 4:3, 155-160 (2012). (in Russian).

26. M. Gürses, A.V. Zhiber, I.T. Habibullin. Characteristic Lie rings of differential equations // Ufimskji Matem. Zhurn. 4:1, 53-62 (2012). (in Russian).

27. A.V. Zhiber, S.N. Kamaeva. Construction of exact solution to sine-Gordon equation on the base of its characteristic Lie ring // Ufimskij Matem. Zhurn. 8:3, 49-58 (2016). [Ufa Math. J. 8:3, 49-57 (2016).]

28. I. Habibullin, M. Poptsova. Classification of a subclass of two-dimensional lattices via characteristic Lie rings // SIGMA. 13:073 (2017).

29. M.N. Poptsova, I.T. Habibullin. Algebraic properties of quasilinear two-dimensional lattices connected with integrability // Ufimskij Matem. Zhurn. 10:3, 89-109 (2018). [Ufa Math. J. 10:3, 86-105 (2018).]

30. I.T. Habibullin, M.N. Kuznetsova. A classification algorithm for integrable two-dimensional lattices via Lie-Rinehart algebras // Teor. Matem. Fiz. 203:1, 161-173 (2020). [Theor. Math. Phys. 203:1, 569-581 (2020).]

31. A.B. Zhiber, R.D. Murtazina, I.T. Habibullin, A.B. Shabat. Equations of mathematical physics. Nonlinear integrable equations. Yurait, Moscow (2018). (in Russian).

32. A.V. Zhiber, A.B. Shabat. Klein-Gordon equations with a nontrivial group // Dokl. AN SSSR. 247:5, 1103-1107 (1979). [Sov. Phys. Dokl. 24:8, 608-609 (1979).]

33. D.V. Millionshchikov. Characteristic Lie algebras of the sinh-Gordon and Tzitzeica equations // Uspekhi Matem. Nauk. 72:6, 203-204 (2017). [Russ. Math. Surv. 72:6, 1174-1176 (2017).]

34. D. Millionshchikov. Lie algebras of slow growth and Klein-Gordon PDE // Algeb. Represent. Theo. 21:5, 1037-1069 (2018). 
35. G. Rinehart. Differential forms for general commutative algebras // Trans. Amer. Math. Soc. 108:2, 195-222 (1963).

36. V.M. Buchstaber, D.V. Leykin. Polynomial Lie algebras // Funk. Anal. Pril. 36:4, 18-34 (2002). [Func. Anal. Appl. 36:4, 267-280 (2002).]

37. D.V. Millionshchikov. Polynomial Lie algebras and growth of their finitely generated Lie subalgebras // Trudy MIAN. 302, 316-333 (2018). [Proc. Steklov Inst. Math. 302, 298-314 (2018).]

38. V.E. Adler, S.Ya. Startsev. Discrete analogues of the Liouville equation // Teor. Mat. Fiz. 121:2, 271-285 (1999). [Theor. Math. Phys. 121:2, 1484-1495 (1999).]

39. V.G. Kac. Simple irreducible graded Lie algebras of finite growth // Izv. AN SSSR. Ser. Matem. 32:5, 1323-1367 (1968). [Math. USSR-Izv. 2:6, 1271-1311 (1968).]

40. O. Mathieu. Classification of simple graded Lie algebras of finite growth // Invent. Math. 108, 455-519 (1990).

41. G.R. Krause, T.H. Lenagan. Growth of algebras and Gelfand-Kirillov dimension. Amer. Math. Soc., Providence, R.I. (2000).

42. I.M. Gelfand, A.A. Kirillov. Sur les corps liés aux algèbres enveloppantes des algèbres de Lie // Inst. Hautes Etudes Sci. Publ. Math. 31, 5-19 (1966).

43. J. Riordan. Combinatorial identities. John Wiley and Sons, New York (1968).

Dmitrii Vladimirovich Millionshchikov,

Lomonosov Moscow State University,

Faculty of Mechanics and Mathematics,

Leninskiye Gory, bld. 1,

119991, Moscow, Russia

Moscow Center of Fundamental and Applied Mathematics,

Leninskiye Gory, bld. 1,

119991, Moscow, Russia

Gubkin University,

National University of Oil and Gas,

Leninsky av. 65,

119991, Moscow, Russia

E-mail: millionshchikov.d@gubkin.ru

Sergey Valerievich Smirnov,

Lomonosov Moscow State University,

Faculty of Mechanics and Mathematics,

Leninskiye Gory, bld. 1,

119991, Moscow, Russia

E-mail: ssmirnov@higeom.math.msu.su 